\title{
Basket Credit Derivative Pricing in a Markov Chain Model with Interacting Intensities
}

\author{
Kangquan Zhi $\mathbb{D}^{1},{ }^{1}$ Jie Guo, ${ }^{2}$ and Xiaosong Qian $^{3}$ \\ ${ }^{1}$ Center for Financial Engineering, Soochow University, Suzhou 215006, China \\ ${ }^{2}$ School of Mathematics and Physics, Suzhou University of Science and Technology, Suzhou 215009, China \\ ${ }^{3}$ School of Mathematical Sciences and Center for Financial Engineering, Soochow University, Suzhou 215006, China \\ Correspondence should be addressed to Kangquan Zhi; zhikangquan96@163.com
}

Received 18 June 2020; Accepted 20 September 2020; Published 17 October 2020

Academic Editor: Wenguang Yu

Copyright (c) 2020 Kangquan Zhi et al. This is an open access article distributed under the Creative Commons Attribution License, which permits unrestricted use, distribution, and reproduction in any medium, provided the original work is properly cited.

\begin{abstract}
In this paper, we propose a Markov chain model to price basket credit default swap (BCDS) and basket credit-linked note (BCLN) with counterparty and contagion risks. Suppose that the default intensity processes of reference entities and the counterparty are driven by a common external shock as well as defaults of other names in the contracts. The stochastic intensity of the external shock is a Cox process with jumps. We derive recursive formulas for the joint distribution of default times and obtain closed-form premium rates for BCDS and BCLN. Numerical experiments are performed to show how the correlated default risks may affect the premium rates.
\end{abstract}

\section{Introduction}

The market for credit derivatives has experienced rapid development during the past decades until the international financial crisis in 2008, which was mainly due to the underestimation of the correlated default risk. Since then, more and more research studies focus on the basket credit derivative pricing. Basket credit default swap (BCDS) and basket credit-linked notes (BCLNs) are two popular multiname credit derivatives, which can reduce the adverse impact of reference assets' defaults on financial institutions. A BCDS is designed to transfer the credit exposure of fixed income products between two parties with $N$ reference entities. The issuer of the contract is the protection seller, and the investor is the protection buyer. A BCDS will have a premium rate and maturity date, and the maturity depends on the performance of reference entities and the counterparty. A BCLN is a note paying an enhanced coupon to investors for bearing the credit risk of $N$ reference entities. The issuer of the note is the protection buyer, and the investor is the protection seller. A BCLN will have a coupon rate, maturity date, and par value just like a standard bond.
However, the maturity depends on the performance of the reference entities and the counterparty.

The reduced-form models are widely used to price credit derivatives. In a reduced-form model, there are mainly three approaches to model the default correlation: copula, conditional independence, and contagion. In the copula approach, the joint distribution of the default times is constructed by combining marginal distributions of the individual by a copula function, see Li [1], Schőnbucher and Schubert [2], Brigo and Capponi [3], and Jean-David [4]. The conditional independence approach assumes that the default intensities are conditionally independent under the given filtration, see Wang and Garleanu [5], Giesecke [6], and Liang et al. [7]. In the contagion approach, the default intensity of one entity is affected not only by systematic factors but also by the default of other entities in the contract, see Jarrow and Yu [8], Zheng and Jiang [9], and Dong and Wang [10].

In this paper, we focus on the pricing of basket credit derivatives (BCDS and BCLN). In the existing literature, different approaches in pricing BCDS and BCLN have been developed. Hull and White [11] developed two fast 
procedures for valuing $k$ th-to-default swaps. Walker [12] studied counterparty risk in BCDS valuation by using a fourstate Markov process that includes contagion effects. Yu [13] gave the Monte Carlo method for pricing basket CDS. Frey and Backhaus [14] considered reduced-form models for portfolio credit risk with interacting default intensities. Zheng and Jiang [9] proposed a factor contagion model for the basket CDS pricing. Wu [15] explored a reasonable coupon rate for basket credit-linked notes (BCLN) with the issuer default risk. Herbertsson et al. [16] valued $k$ th-todefault swap spreads in a tractable shot noise model. Wang et al. [17] proposed a model for pricing a basket CDS with the negative correlation between prepayment and default under the bottom-up framework. Li and Li [18] used a type of dynamic copula method to characterize the dependence structure between financial assets and price basket default swaps. Esfahanipour and Jahanbin [19] proposed a heuristic algorithm for pricing of basket default swaps. Dong et al. [20] studied the $k$ th-to-default basket swap under a correlated regime-switching hazard process model. Guo et al. [21] employed an intensity-based credit risk model with regime switching to consider the valuation of basket CDS in a homogeneous portfolio. The previous work did not combine internal contagion and external shock, which is a Cox process. In addition, they seldom obtained the closed-form solutions. In this paper, we propose a model that combines internal contagion and random external shock. Our model is not only applicable to BCDS pricing but also to BCLN. Furthermore, we derive the closed-form solution which is not easy for the complex structure of the default as the numerical analysis can be done very smoothly.

Leung and Kwok [22] presented a Markov chain model to price single-name CDS; the default intensities of the reference entity and counterparty were affected by an external shock. Inspired by Leung and Kwok [22], we present a more general model to study basket credit derivatives with interacting default intensities, which are driven by an external shock as well as defaults of other names in the contracts. We get recursive formulas for the unconditional distribution of default times through ingenious construction of the infinitesimal generator matrix and obtain closed-form premium rates for basket credit default swap and basket credit-linked notes.

The paper is organized as follows. In Section 2, we give the construction of interacting default intensities and derive the joint distribution of default times by solving a system of ordinary differential equations. In Section 3, we calculate the premium rates of $k$ th-to-default CDS and $k$ th-to-default CLN with the counterparty risk. Numerical results are presented to show how the correlated default risks affect the premium rates in Section 4. At last, we conclude the paper.

\section{Markov Chain Model with Interacting Intensities}

In this section, we construct a reduced-form model with stochastic default intensities by a Markov chain. Consider a complete filtered probability space $\left(\Omega, \mathscr{G},\left\{\mathscr{G}_{t}\right\}_{0 \leq t \leq T}, P\right)$, where $P$ is a martingale measure and $\left\{\mathscr{G}_{t}\right\}_{t \geq 0}$ is filtration satisfying the usual conditions. Our model includes a basket credit derivative and an external shock. The basket credit derivative includes $N$ reference entities, a counterparty, and an investor. Let $R_{i}$ represent the $i$ th reference entity with default time $\tau_{R_{i}}$ for $i \in I=\{1,2, \ldots, N\}, C$ be the counterparty with default time $\tau_{C}, S$ be the external shock with arrival time $\tau_{S}$, and $\mathscr{L}=\left\{R_{1}, R_{2}, \ldots, R_{N}, C, S\right\}$ be the set which contains all of the names in the model. Assume that $\tau_{S}$ is independent of $\tau_{C}$ and $\tau_{R_{i}}(i \in I)$. The default process of our model is given by

$$
\mathbf{H}_{t}=\left(H_{t}^{R_{1}}, H_{t}^{R_{2}}, \ldots, H_{t}^{R_{N}}, H_{t}^{C}, H_{t}^{S}\right),
$$

where

$$
\left\{\begin{array}{l}
H_{t}^{R_{i}}=\mathbf{1}_{\left\{\tau_{R_{i}} \leq t\right\}}, \quad i \in I, \\
H_{t}^{C}=\mathbf{1}_{\left\{\tau_{C} \leq t\right\}}, \\
H_{t}^{S}=\mathbf{1}_{\left\{\tau_{S} \leq t\right\}},
\end{array}\right.
$$

and $\mathbf{1}_{\{\tau \leq t\}}$ is the indicator function. $\mathbf{H}_{t}$ is a finite-state Markov chain with state space $O=\{0,1\}^{N+2}$.

The macroeconomic variables are described by a stochastic process $\Psi=\left(\Psi_{t}\right)_{0 \leq t \leq T}$. An investor can get the historical information of the macroeconomic variables and the default status of all names in our model at time $t$. The filtration $\left(\mathscr{G}_{t}\right)_{t \geq 0}$ is given by

$$
\mathscr{G}_{t}=\mathscr{F}_{t}^{\Psi} \vee \mathscr{H}_{t}^{R_{1}} \vee \mathscr{H}_{t}^{R_{2}} \vee \cdots \vee \mathscr{H}_{t}^{R_{N}} \vee \mathscr{H}_{t}^{C} \vee \mathscr{H}_{t}^{S} \text {, }
$$

where

$$
\left\{\begin{array}{l}
\mathscr{F}_{t}^{\Psi}=\sigma\left(\Psi_{s}: 0 \leq s \leq t\right), \\
\mathscr{H}_{t}^{C}=\sigma\left(H_{s}^{C}: 0 \leq s \leq t\right), \\
\mathscr{H}_{t}^{S}=\sigma\left(H_{s}^{S}: 0 \leq s \leq t\right), \\
\mathscr{H}_{t}^{R_{i}}=\sigma\left(H_{s}^{R_{i}}: 0 \leq s \leq t\right), \quad i \in I,
\end{array}\right.
$$

and $G_{t}$ is the $\sigma$-field generated by $\mathscr{F}_{t}^{\Psi} \cup \mathscr{H}_{t}^{R_{1}} \cup \mathscr{H}_{t}^{R_{2}} \cup \cdots \cup$ $\mathscr{H}_{t}^{R_{N}} \cup \mathscr{H}_{t}^{C} \cup \mathscr{H}_{t}^{S}$. The martingale default intensities of the reference entities and counterparty are, respectively, defined by $\lambda^{R_{i}}\left(\Psi_{t}, \mathbf{H}_{t}\right)(i \in I)$ and $\lambda^{C}\left(\Psi_{t}, \mathbf{H}_{t}\right)$, which satisfy the property that

$$
\left\{\begin{array}{l}
H_{t}^{C}-\int_{0}^{t \wedge \tau_{C}} \lambda^{C}\left(\Psi_{s}, \mathbf{H}_{s}\right) \mathrm{d} s, \\
H_{t}^{R_{i}}-\int_{0}^{t \wedge \tau_{R_{i}}} \lambda^{R_{i}}\left(\Psi_{s}, \mathbf{H}_{s}\right) \mathrm{d} s, \quad i \in I,
\end{array}\right.
$$

are $\left\{\mathscr{G}_{t}\right\}$-martingales.

The arrival of the external shock is modeled by a Cox process with stochastic intensity $\lambda_{t}^{S}$. Before the shock $S$ happens, the default intensities of $\lambda_{t}^{R_{i}} \quad(i \in I)$ and $\lambda_{t}^{C}$ are, respectively, assumed to be $a_{t}^{R_{i}}$ and $a_{t}^{C}$, where $a_{t}^{R_{i}}$ and $a_{t}^{C}$ are some deterministic functions of $t$. When the shock $S$ happens, $\lambda_{t}^{R_{i}}$ and $\lambda_{t}^{C}$ jump to $\alpha_{R_{i}}^{S} a_{t}^{R_{i}}$ and $\alpha_{C}^{S} a_{t}^{C}$, respectively, where nonnegative constants $\alpha_{R_{i}}^{S}$ and $\alpha_{C}^{S}$ denote the effects from the external shock to reference entities and the counterparty. Depending on whether $\alpha_{R_{i}}^{S}$ and $\alpha_{C}^{S}$ are greater 
than 1 or not, the densities $\lambda^{R_{i}}$ and $\lambda^{C}$ can jump upward or downward. Besides, two kinds of contagion risks are considered in our model. One is the contagion effects between reference entities, and the other one is the contagion effects from reference entities to the counterparty. If reference entity $i$ defaults, the default intensities of other reference entities and the counterparty jump to $\alpha_{R_{j}}^{R_{i}} a_{t}^{R_{j}} \quad(j \neq i)$ and $\alpha_{C}^{R_{i}} a_{t}^{C}$, respectively, where $\alpha_{R_{j}}^{R_{i}}$ and $\alpha_{C}^{R_{i}}$ are nonnegative constants. We do not consider the contagion effects from the counterparty to reference entities because the credit derivative would be terminated if the counterparty defaulted first. In summary, the default intensities of reference entities and the counterparty can be expressed as follows:

$$
\begin{gathered}
\lambda_{\mathrm{t}}^{R_{i}}=a_{t}^{R_{i}}\left[\left(\alpha_{R_{i}}^{S}-1\right) I_{\tau_{S} \leq t}+1\right] \prod_{j \in I \backslash\{i\}}\left[\left(\alpha_{R_{i}}^{R_{j}}-1\right) I_{\tau_{R_{j}} \leq t}+1\right], \\
\alpha_{R_{i}}^{S}, \alpha_{R_{i}}^{R_{j}} \geq 0, i, j \in I \text { and } i \neq j, \\
\lambda_{\mathrm{t}}^{C}=a_{t}^{C}\left[\left(\alpha_{C}^{S}-1\right) I_{\tau_{S} \leq t}+1\right] \prod_{i \in I}\left[\left(\alpha_{C}^{R_{i}}-1\right) I_{\tau_{R_{i}} \leq t}+1\right], \\
\alpha_{C}^{S}, \alpha_{C}^{R_{i}} \geq 0, i \in I .
\end{gathered}
$$

We assume that the simultaneous defaults or shock cannot happen in the model for the sake of simplicity. In this paper, we consider basket credit derivatives with $N$ reference entities, and the state space $O$ of $\mathbf{H}_{t}$ is $2^{N+2}$ dimensions. Let $\psi^{S}=\left(\lambda_{t}^{S}\right)_{t \in[0, T]}$, and conditional on the given state $\psi^{S}$, the infinitesimal generator matrix of $\mathbf{H}_{t}$ is $\Lambda_{\left[\psi^{s}\right]}(t) \triangleq\left(\Lambda_{\mathrm{H}^{\prime} \mathrm{H}^{\prime}}(t) \mid \psi^{S}\right)_{2^{N+2} \times 2^{N+2}}$, where $\mathrm{H}, \mathrm{H}^{\prime}=\left(H^{R_{1}}\right.$, $\left.\ldots, H^{R_{N}}, H^{C}, H^{S}\right) \in$ Orepresent the states of the Markov chain $\mathbf{H}_{t}$. The conditional transition probability matrix $P\left(0, t \mid \psi^{S}\right) \triangleq\left(P_{\mathrm{H} \times \mathrm{H}^{\prime}}\left(0, t \mid \psi^{S}\right)\right)_{2^{N+2} \times 2^{N+2}}$ is governed by the following forward Kolmogorov equation:

$$
\frac{\mathrm{d} P\left(0, t \mid \psi^{S}\right)}{\mathrm{d} t}=P\left(0, t \mid \psi^{S}\right) \Lambda_{\left[\psi^{S}\right]}(t), \quad t \geq 0,
$$

with $P\left(0,0 \mid \psi^{S}\right)=E$, and $E$ is the unit matrix. Because the default states and the shock are absorbing states for the Markov chain $\mathbf{H}_{t}$, the matrix $\Lambda_{\left[\psi^{s}\right]}(t)$ is upper triangular, and the conditional transition probabilities $P\left(0, t \mid \psi^{S}\right)$ can be solved successively in a sequential manner.

To describe the generator matrix $\Lambda_{\left[\psi^{s}\right]}(t)=$ $\left(\Lambda_{\mathrm{H} \times \mathrm{H}^{\prime}}(t) \mid \psi^{S}\right)_{2^{N+2} \times 2^{N+2}}$, we introduce some auxiliary notations as follows:

(i) $\mathrm{H}^{R_{M}}$ represents the state that only reference entities in set $M \subset I$ defaulted. Here,
$\mathrm{H}^{R_{M}}=\left(H^{R_{1}}, \ldots\right.$,

$\left.H^{R_{N}}, H^{C}, H^{S}\right)=(\sim 0, \ldots, 1, \ldots, 0,0,0), \quad$ where $H^{R_{i}}={ }_{n} 1$ for all $i \in M$. Especially, $\mathrm{H}^{R_{\phi}}=$ $(\sim 0, \ldots, 0,0,0)$ represents the state that no reference entity defaults.

(ii) $\mathrm{H}^{C}$ represents the state that only the counterparty defaulted.

Here, $H^{C}=\left(H^{R_{1}}, \ldots, H^{R_{N}}, H^{C}, H^{S}\right)=(\sim 0, \ldots$, $0,1,0)$.

(iii) $\mathrm{H}^{S}$ represents the state that only the external shock arrived.

Here, $\mathrm{H}^{S}=\left(H^{R_{1}}, \ldots, H^{R_{N}}, H^{C}, H^{S}\right)=(\sim 0, \ldots$, $0,0,1)$.

(iv) $\mathrm{H}^{R_{M} \cup C}$ represents the state that reference entities in set $M \subset I$ and the counterparty $C$ defaulted.

Here, $\mathrm{H}^{R_{M} \cup C}=\left(H^{R_{1}}, \ldots, H^{R_{N}}, H^{C}, H^{S}\right)=(\stackrel{N}{\sim} 0$, $\ldots, 1, \ldots, 0,1,0)$, where $H^{R_{i}}=1$ for all $i \in M$.

(v) $\mathrm{H}^{R_{M} \cup S}$ represents the state that reference entities in set $M \subset I$ defaulted, and the external shock $S$ happened.

Here, $\mathrm{H}^{R_{M} \cup S}=\left(H^{R_{1}}, \ldots, H^{R_{N}}, H^{C}, H^{S}\right)=(\stackrel{N}{\sim} 0$, $\ldots, 1, \ldots, 0,0,1)$, where $H^{R_{i}}=1$ for all $i \in M$.

(vi) $\mathrm{H}^{R_{M} \cup C \cup S}$ represents the state that reference entities in set $M \subset I$ and the counterparty $C$ both defaulted, and the external shock $S$ happened.

Here, $\quad \mathrm{H}^{R_{M} \cup C \cup S}=\left(H^{R_{1}}, \ldots, \quad H^{R_{N}}, H^{C}, H^{S}\right)=$ $(\sim 0, \ldots, 1, \ldots, 0,1,1)$, where $H^{R_{i}}=1$ for all $i \in M$.

(vii) $\Lambda_{\mathrm{H}}(t) \triangleq \Lambda_{\mathrm{H} \times \mathrm{H}}(t)$ for $\mathrm{H} \in O$ are elements on the diagonal of the matrix $\Lambda_{\left[\psi^{s}\right]}(t)$, which represent the intensities that the Markov chain $\mathbf{H}_{t}$ remains at states $\mathrm{H}$.

(viii) $P_{\mathrm{H}}\left(0, t \mid \psi^{S}\right) \triangleq P_{\mathrm{H}^{R}{ } \times \mathrm{H}}\left(0, t \mid \psi^{S}\right) \quad$ represents the conditional probability that Markov chain $\mathbf{H}_{t}$ transfers from the initial state $\mathrm{H}^{R_{\phi}}$ to state $\mathrm{H}$ during $[0, t]$. It is the first line of the conditional transition probability matrix $P\left(0, t \mid \psi^{S}\right)$ and can be used to present the joint distributions of default times during $[0, t]$.

Firstly, we calculate the conditional transition probabilities that none of the reference entities defaults during $[0, t]$. The corresponding states of $\mathbf{H}_{t}$ are $\mathrm{H}^{R_{\phi}}$ and $\mathrm{H}^{S}$. The corresponding elements in the infinitesimal generator matrix $\Lambda_{\left[\psi^{s}\right]}(t)$ are as follows:

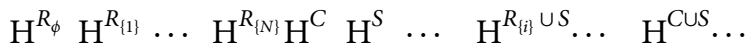

$$
\begin{aligned}
& \begin{array}{c}
\mathrm{H}^{R_{\phi}} \\
\vdots \\
\mathrm{H}^{S} \\
\vdots
\end{array} \quad\left(\begin{array}{ccccccccccc}
\Lambda_{\mathrm{H}^{R_{\phi}}} & a_{t}^{R_{1}} & \cdots & a_{t}^{R_{N}} & a_{t}^{C} & \lambda_{t}^{S} & \cdots & 0 & \cdots & 0 & \cdots \\
\vdots & \vdots & \vdots & \vdots & \vdots & \vdots & \vdots & \vdots & \vdots & \vdots & \vdots \\
0 & 0 & \cdots & 0 & 0 & \Lambda_{\mathrm{H}^{S}} & \cdots & \alpha_{R_{i}}^{S} a_{t}^{R_{i}} & \cdots & \alpha_{C}^{S} a_{t}^{C} & \cdots \\
\vdots & \vdots & \vdots & \vdots & \vdots & \vdots & \vdots & \vdots & \vdots & \vdots & \vdots
\end{array}\right) .
\end{aligned}
$$


In the $\mathrm{H}^{R_{\varnothing}}$-row of the generator matrix $\Lambda_{\left[\psi^{s}\right]}(t)$, the positive elements represent the transition intensities from state $\mathrm{H}^{R_{\varnothing}}$ to other states by one jump. Because the cases of simultaneous defaults or shock are not examined here, the possible positive elements in this row are $a_{t}^{R_{i}}(i \in I), a_{t}^{C}$, or $\lambda_{t}^{S}$. Noting that the elements on the diagonal of the generator matrix $\Lambda_{\left[\psi^{s}\right]}(t)$ are the sum of all other elements in the row multiplied with -1 , we can get the first element $\Lambda_{\mathrm{H}^{R_{\phi}}}$ of the $\mathrm{H}^{R_{\varnothing}}$-row as follows:

$$
\Lambda_{\mathrm{H}^{R_{\phi}}}(t)=-\left[\sum_{i \in I} a_{t}^{R_{i}}+a_{t}^{C}+\lambda_{t}^{S}\right] .
$$

Because the generator matrix $\Lambda_{\left[\psi^{s}\right]}(t)$ is upper diagonal, $\Lambda_{\mathrm{H}^{R_{\phi}}}(t)$ is the only nonzero element in the $\mathrm{H}^{R_{\phi}}$-column.

In the $\mathrm{H}^{S}$-row of the generator matrix $\Lambda_{\left[\psi^{S}\right]}(t)$, the positive elements represent the transition intensities from state $\mathrm{H}^{S}$ to other states by one jump. By (6), the positive elements in the $\mathrm{H}^{S}$-row are $\alpha_{R_{i}}^{S} a_{t}^{R_{i}}(i \in I)$ and $\alpha_{C}^{S} a_{t}^{C}$. Summing these positive elements and multiplying the sum with -1 , we get the diagonal element in this row as follows:

$$
\Lambda_{\mathrm{H}^{S}}(t)=-\left[\sum_{i \in I} \alpha_{R_{i}}^{S} a_{t}^{R_{i}}+\alpha_{C}^{S} a_{t}^{C}\right] .
$$

In the $\mathrm{H}^{S}$-column of the generator matrix $\Lambda_{\left[\psi^{S}\right]}(t)$, the positive elements represent the transition intensities from other states to state $\mathrm{H}^{S}$ by one jump. The only possible state which can jump to $\mathrm{H}^{S}$ is $\mathrm{H}^{R_{\phi}}$. So, the nonzero elements in the $\mathrm{H}^{S}$-column are $\lambda_{t}^{S}$ and $\Lambda_{\mathrm{H}^{s}}(t)$.

By forward Kolmogorov equation (7), we have

$$
\left\{\begin{array}{l}
\frac{\mathrm{d} P_{\mathrm{H}^{R_{\phi}}}\left(0, t \mid \psi^{S}\right)}{\mathrm{d} t}=P_{\mathrm{H}^{R_{\phi}}}\left(0, t \mid \psi^{S}\right) \Lambda_{\mathrm{H}^{R_{\phi}}}(t), \\
\frac{\mathrm{d} P_{\mathrm{H}^{S}}\left(0, t \mid \psi^{S}\right)}{\mathrm{d} t}=P_{\mathrm{H}^{R_{\phi}}}\left(0, t \mid \psi^{S}\right) \lambda_{t}^{S}+P_{\mathrm{H}^{S}}\left(0, t \mid \psi^{S}\right) \Lambda_{\mathrm{H}^{S}}(t) .
\end{array}\right.
$$
follows:

It is easy to obtain the solutions of the above equations as

$$
\left\{\begin{array}{l}
P_{\mathrm{H}^{R_{\phi}}}\left(0, t \mid \psi^{S}\right)=e^{\int_{0}^{t} \Lambda_{\mathrm{H}^{R_{\phi}}}(u) \mathrm{d} u} \\
P_{\mathrm{H}^{S}}\left(0, t \mid \psi^{S}\right)=e^{\int_{0}^{t} \Lambda_{\mathrm{H}^{S}}(u) \mathrm{d} u} \int_{0}^{t} \lambda_{u}^{S} e^{-\int_{0}^{u} \Lambda_{\mathrm{H}^{S}}(r) \mathrm{d} r} P_{\mathrm{H}^{R_{\phi}}}\left(0, u \mid \psi^{S}\right) \mathrm{d} u .
\end{array}\right.
$$

Secondly, we calculate the conditional transition probabilities that only one reference entity defaults during $[0, t]$. The corresponding states of $\mathbf{H}_{t}$ are $\mathrm{H}^{R_{\{i\}}}$ and $\mathrm{H}^{R_{\{i\}} \cup S}$ for $i \in I$. The corresponding elements in the infinitesimal generator matrix $\Lambda_{\left[\psi^{s}\right]}(t)$ are as follows:

$$
\begin{aligned}
& \begin{array}{lllllllllllllll}
\cdots & \mathrm{H}^{R_{\{i\}}} & \cdots & \mathrm{H}^{R_{\{i, j\}}} & \cdots & \mathrm{H}^{R_{(i)} \cup C} & \ldots & \mathrm{H}^{R_{(i)} \cup S} & \cdots & \mathrm{H}^{R_{(i, j)} \cup S} & \cdots & \mathrm{H}^{R_{(i)}} \cup C \cup S & \ldots
\end{array}
\end{aligned}
$$

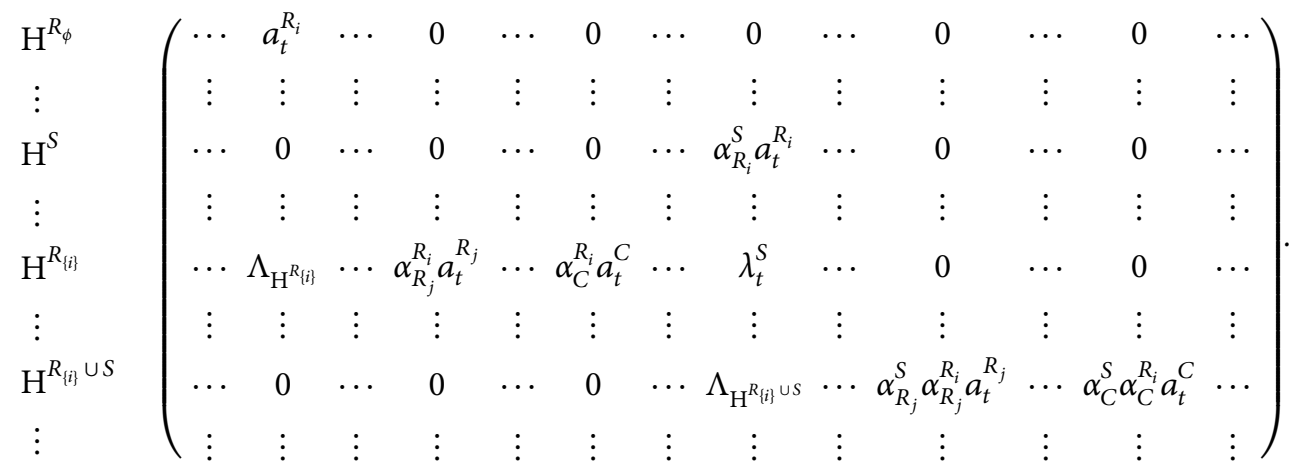

In the $\mathrm{H}^{R_{\{i\}}}$-row of the generator matrix $\Lambda_{\left[\psi^{s}\right]}(t)$, the positive elements represent the transition intensities from state $\mathrm{H}^{R_{\{i\}}}$ to other states by one jump. There are three possible scenarios: a single reference entity defaults, counterparty defaults, or external shock happens. The positive elements in this row are $\alpha_{R_{j}}^{R_{i}} a_{t}^{R_{j}}(j \in I \backslash\{i\})$ and $\alpha_{C}^{R_{i}} a_{t}^{C}$ by (6) and $\lambda_{t}^{S}$ if external shock happens. The diagonal element in this row is as follows:

$$
\Lambda_{\mathrm{H}^{R i\}}}(t)=-\left[\sum_{j \subset I \backslash\{i\}} \alpha_{R_{j}}^{R_{i}} a_{t}^{R_{j}}+\alpha_{C}^{R_{i}} a_{t}^{C}+\lambda_{t}^{S}\right] .
$$

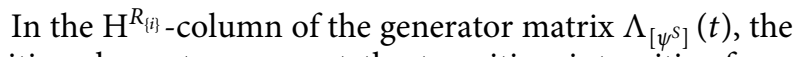
positive elements represent the transition intensities from other states to state $\mathrm{H}^{R_{i i\}}}$ by one jump. The only possible state which can jump to $\mathrm{H}^{R_{\{i\}}}$ is $\mathrm{H}^{R_{\phi}}$. So, the nonzero elements in the $\mathrm{H}^{R_{i}}$-column are $a_{t}^{R_{i}}$ and the diagonal element $\Lambda_{\mathrm{H}^{R_{i j}}}(t)$.

In the $\mathrm{H}^{R_{\{i\}} \cup S}$-row of the generator matrix $\Lambda_{\left[\psi^{S}\right]}(t)$, the positive elements represent the transition intensities from state $\mathrm{H}^{R_{\{i\}}} \cup S$ to other states by one jump. There are two possible scenarios: a single reference entity defaults or counterparty defaults. By (6), the positive elements in this row are $\alpha_{R_{j}}^{S} \alpha_{R_{j}}^{R_{i}} a_{t}^{R_{j}}(j \in I \backslash\{i\})$ and $\alpha_{C}^{S} \alpha_{C}^{R_{i}} a_{t}^{C}$. The diagonal element in this row is as follows:

$$
\Lambda_{\mathrm{H}^{R_{\{i\}} \cup s}}(t)=-\left[\sum_{j \in I \backslash\{i\}} \alpha_{R_{j}}^{S} \alpha_{R_{j}}^{R_{i}} a_{t}^{R_{j}}+\alpha_{C}^{S} \alpha_{C}^{R_{i}} a_{t}^{C}\right] .
$$


In the $\mathrm{H}^{R_{i i} \cup S}$-column of the generator matrix $\Lambda_{\left[\psi^{S}\right]}(t)$, the positive elements represent the transition intensities from other states to state $\mathrm{H}^{R_{i j}} \cup S$ by one jump. The possible states which can jump to $\mathrm{H}^{R_{i i}} \cup S$ are $\mathrm{H}^{R_{i i\}}}$ and $\mathrm{H}^{S}$. So, the nonzero elements in the $\mathrm{H}^{R_{i i\}} \cup S_{-}}$-column are $\alpha_{R_{i}}^{S} a_{t}^{R_{i}}, \lambda_{t}^{S}$, and the diagonal element $\Lambda_{\mathrm{H}^{R}\{i\}} \cup$.

By forward Kolmogorov equation (7), we have

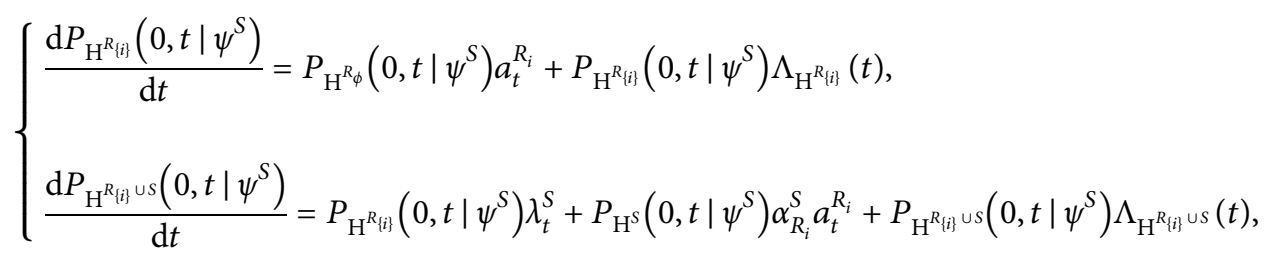

where $P_{\mathrm{H}^{R_{\phi}}}\left(0, t \mid \psi^{S}\right)$ and $P_{\mathrm{H}^{S}}\left(0, t \mid \psi^{S}\right)$ are already obtained

in (12). It is easy to solve equation (16) as follows:

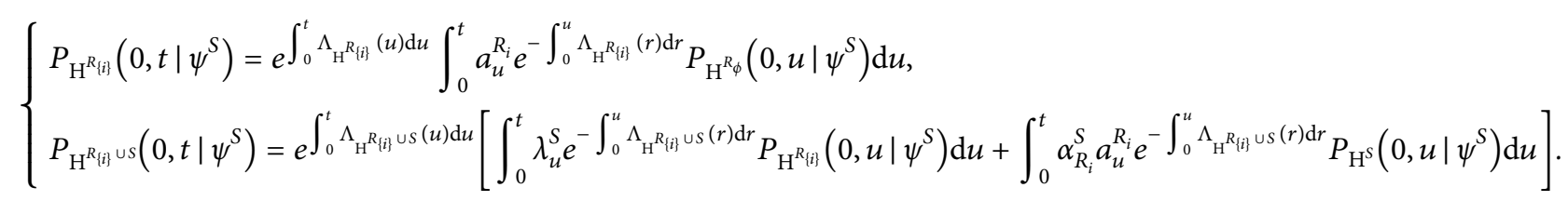

We can derive all the conditional transition probabilities by the method of mathematical induction. Suppose that we have obtained the conditional transition probabilities that $k-1$ reference entities default during $[0, t]$. For the cases that $k$ reference entities default, the corresponding states of $\mathbf{H}_{t}$ are $\mathrm{H}^{R_{M}}$ and $\mathrm{H}^{R_{M}} \cup S$ for $M \subset I$ and $|M|=k$, where $|M|$ is the cardinal number of set $M$. The corresponding elements in the infinitesimal generator matrix $\Lambda_{\left[\psi^{s}\right]}(t)$ are as follows:

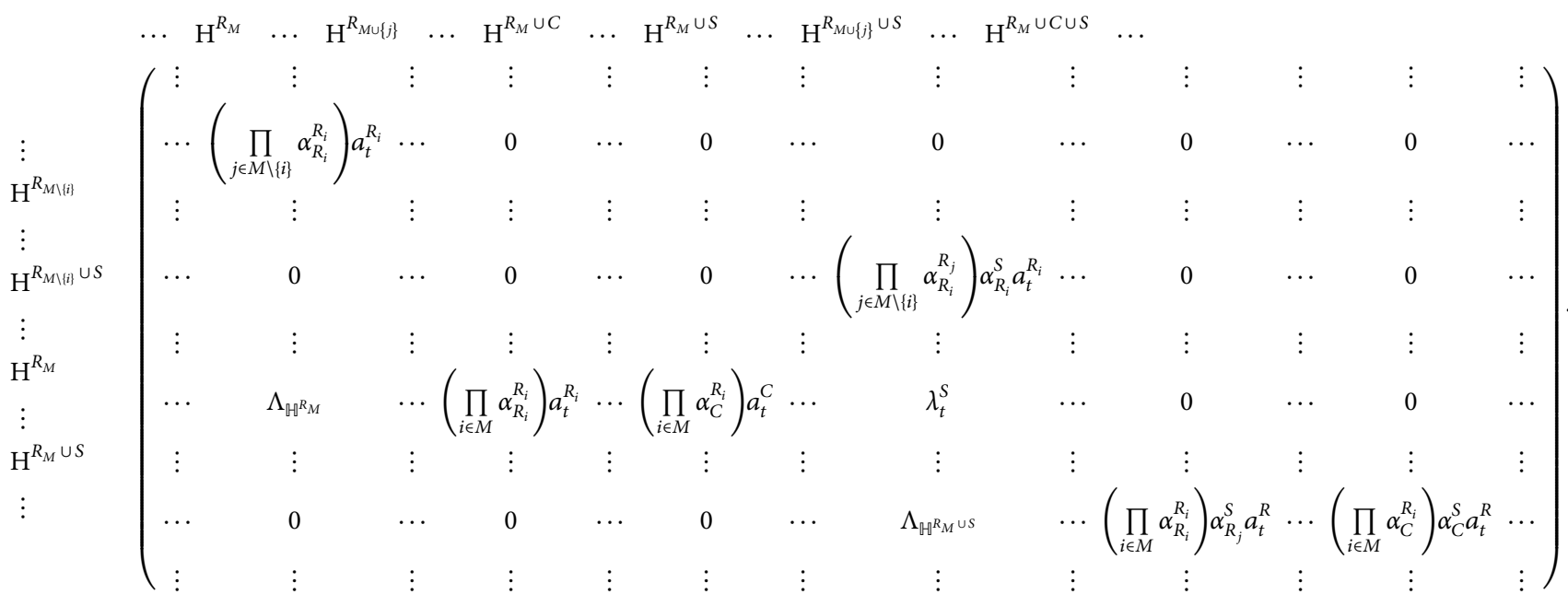

In the $\mathrm{H}^{R_{M}}$-row of the generator matrix $\Lambda_{\left[\psi^{s}\right]}(t)$, the positive elements represent the transition intensities from state $\mathrm{H}^{R_{M}}$ to other states by one jump. There are three possible scenarios: a single reference entity defaults, counterparty defaults, or external shock happens. The positive elements in this row are $\left(\prod_{i \in M} \alpha_{R_{i}}^{R_{i}}\right) a_{t}^{R_{j}}(j \in I \backslash M)$ and $\left(\prod_{i \in M} \alpha_{C}^{R_{i}}\right) a_{t}^{C}$ by (7) and $\lambda_{t}^{S}$ if external shock happens. The diagonal element in this row is as follows: 


$$
\Lambda_{\mathrm{H}^{R_{M}}}(t)=-\left[\sum_{j \in I \backslash M}\left(\prod_{i \in M} \alpha_{R_{j}}^{R_{i}}\right) a_{t}^{R_{j}}+\left(\prod_{i \in M} \alpha_{C}^{R_{i}}\right) a_{t}^{C}+\lambda_{t}^{S}\right] .
$$

In the $\mathrm{H}^{R_{M}}$-column of the generator matrix $\Lambda_{\left[\psi^{s}\right]}(t)$, the positive elements represent the transition intensities from other states to state $\mathrm{H}^{R_{M}}$ by one jump. The possible states which can jump to $\mathrm{H}^{R_{M}}$ are $\mathrm{H}^{R_{M \backslash i\}}}$ for $i \in M$. So, the nonzero elements in this column are $\left(\prod_{j \in M \backslash\{i\}} \alpha_{R_{i}}^{R_{j}}\right) a_{t}^{R_{i}}$ by (6) and the diagonal element $\Lambda_{\mathrm{H}^{R_{M}}}$.

In the $\mathrm{H}^{R_{M} \cup S}$-row of the generator matrix $\Lambda_{\left[\psi^{S}\right]}(t)$, the positive elements represent the transition intensities from state $\mathrm{H}^{R_{M} \cup S}$ to other states by one jump. There are two possible scenarios: a single reference entity defaults or counterparty defaults. By (6), the positive elements in this row are $\left(\prod_{i \in M} \alpha_{R_{j}}^{R_{i}}\right) \alpha_{R_{j}}^{S} a_{t}^{R_{j}}(j \in I \backslash M)$ and $\left(\prod_{i \in M} \alpha_{C}^{R_{i}}\right) \alpha_{C}^{S} a_{t}^{C}$. The diagonal element in this row is obtained as follows:

$$
\Lambda_{\mathrm{H}^{R_{M}} \cup S}(t)=-\left[\sum_{j \in I \backslash M}\left(\prod_{i \in M} \alpha_{R_{j}}^{R_{i}}\right) \alpha_{R_{j}}^{S} a_{t}^{R_{j}}+\left(\prod_{i \in M} \alpha_{C}^{R_{i}}\right) \alpha_{C}^{S} a_{t}^{C}\right] .
$$

In the $\mathrm{H}^{R_{M} \cup S}$-column of the generator matrix $\Lambda_{\left[\psi^{S}\right]}(t)$, the positive elements represent the transition intensities from other states to state $\mathrm{H}^{R_{M} \cup S}$ by one jump. The possible states which can jump to $\mathrm{H}^{R_{M} \cup S}$ are $\mathrm{H}^{R_{M}}$ and $\mathrm{H}^{R_{M \backslash\{i\}}} \cup S(i \in M)$. So ${ }_{\vec{R}}$ the nonzero elements in this column are $\lambda_{t}^{S}, \quad\left(\prod_{j \in M \backslash\{i\}} \alpha_{R_{i}}\right) \alpha_{R_{i}}^{S} a_{t}^{R_{i}}$, and the diagonal element $\Lambda_{\mathrm{H}^{R} M} \cup \mathrm{s}$

By forward Kolmogorov equation (7), we have

$$
\left\{\begin{array}{l}
\frac{\mathrm{d} P_{\mathrm{H}^{R_{M}}}\left(0, t \mid \psi^{S}\right)}{\mathrm{d} t}=\sum_{i \in M}\left[P_{\mathrm{H}^{\left.R_{M} \backslash i\right\}}}\left(0, t \mid \psi^{S}\right)\left(\prod_{j \in M \backslash\{i\}} \alpha_{R_{i}}^{R_{j}}\right) a_{t}^{R_{i}}\right]+P_{\mathrm{H}^{R_{M}}}\left(0, t \mid \psi^{S}\right) \Lambda_{\mathrm{H}^{R_{M}}}(t), \\
\frac{\mathrm{d} P_{\mathrm{H}^{R_{M}} \cup s}\left(0, t \mid \psi^{S}\right)}{\mathrm{d} t}=\sum_{i \in M}\left[P_{\mathrm{H}^{\left.R_{M} \backslash i\right\}}} \cup s\left(0, t \mid \psi^{S}\right)\left(\prod_{j \in M \backslash\{i\}} \alpha_{R_{i}}^{R_{j}}\right) \alpha_{R_{i}}^{S} a_{t}^{R_{i}}\right]+P_{\mathrm{H}^{R_{M}}}\left(0, t \mid \psi^{S}\right) \lambda_{t}^{S}+P_{\mathrm{H}^{R_{M}} \cup s}\left(0, t \mid \psi^{S}\right) \Lambda_{\mathrm{H}^{R_{M}} \cup s}(t),
\end{array}\right.
$$

where $P_{\mathrm{H}^{R_{M \backslash\{i\}}}}\left(0, t \mid \psi^{S}\right)$ and $P_{\mathrm{H}^{R} M \backslash\{i\}} \cup S\left(0, t \mid \psi^{S}\right)$ are known by induction. It is easy to get the solutions of equation (21) as follows:

$$
\left\{\begin{array}{l}
P_{\mathrm{H}^{R_{M}}}\left(0, t \mid \psi^{S}\right)=e^{\int_{0}^{t} \Lambda_{\mathrm{H}^{R_{M}}}(u) \mathrm{d} u} \sum_{i \in M}\left[\int_{0}^{t}\left(\prod_{j \in M \backslash\{i\}} \alpha_{R_{i}}^{R_{j}}\right) a_{u}^{R_{i}} e^{-\int_{0}^{u} \Lambda_{\mathrm{H}^{R_{M}}}(r) \mathrm{d} r} \times P_{\mathrm{H}^{\left.R_{M} \backslash i\right\}}}\left(0, u \mid \psi^{S}\right)\right] \mathrm{d} u, \\
P_{\mathrm{H}^{R_{M}} \cup s}\left(0, t \mid \psi^{S}\right)=e^{\int_{0}^{t} \Lambda_{\mathrm{H}^{R_{M}} \cup S}(u) \mathrm{d} u}\left\{\int_{0}^{t} \lambda_{u}^{S} e^{-\int_{0}^{u} \Lambda_{\mathrm{H}^{R_{M}} \cup S}(r) \mathrm{d} r} P_{\mathrm{H}^{R_{M}}}\left(0, u \mid \psi^{S}\right) \mathrm{d} u\right. \\
\left.+\sum_{i \in M}\left[\alpha_{R_{i}}^{S}\left(\prod_{j \in M \backslash\{i\}} \alpha_{R_{i}}^{R_{j}}\right) \int_{0}^{t} a_{u}^{R_{i}} e^{-\int_{0}^{u} \Lambda_{\mathrm{H}^{R_{M}} \cup S}(r) \mathrm{d} r} \times P_{\mathrm{H}^{R_{M} \backslash\{i\}}} \cup s\left(0, u \mid \psi^{S}\right) \mathrm{d} u\right]\right\} .
\end{array}\right.
$$

In order to calculate the unconditional transition probabilities, we need the following proposition.

\section{Proposition 1}

(i) $P_{H^{R_{M}}}\left(0, t \mid \psi^{S}\right)$ contains only one random term $e^{-\int_{0}^{t} \lambda_{u}^{S} d u}$ which is outside the integral symbol; (ii) each term in $P_{H^{R_{M}} \cup S}\left(0, t \mid \psi^{S}\right)$ contains only one random term $\lambda_{u}^{S} e^{-\int_{0}^{u} \lambda_{r}^{S} d r}$ which is inside the integral symbol.

Proof. We use the induction method to prove this proposition.

(i) Firstly, $|M|=0$ :

$$
\begin{aligned}
P_{\mathrm{H}^{R_{\phi}}}\left(0, t \mid \psi^{S}\right) & =e^{\int_{0}^{t} \Lambda_{\mathbb{M}^{R_{\phi}}}(u) \mathrm{d} u} \\
& =e^{-\int_{0}^{t} \lambda_{u}^{S} \mathrm{~d} u} e^{-\int_{0}^{t}\left(\sum_{i \in I} a_{u}^{R_{i}}+a_{u}^{C}\right) \mathrm{d} u} .
\end{aligned}
$$

$P_{\mathrm{H}^{R_{\phi}}}\left(0, t \mid \psi^{S}\right)$ just contains one random term owning the path of $\lambda_{t}^{S}$.

Secondly, $|M|=1$ : 


$$
\begin{aligned}
P_{\mathrm{H}^{R_{\{i\}}}\left(0, t \mid \psi^{S}\right)=} & \int_{0}^{t} \Lambda_{\mathrm{H}}^{R_{\{i\}}(u) \mathrm{d} u} \int_{0}^{t} a_{u}^{R_{i}} e^{-\int_{0}^{u} \Lambda_{\mathrm{H}} R_{\{i\}}(r) \mathrm{d} r} P_{\mathrm{H}^{R_{\phi}}}\left(0, u \mid \psi^{S}\right) \mathrm{d} u \\
= & e^{-\int_{0}^{t}\left(\sum_{j \subset I \backslash\{i\}} \alpha_{R_{j}}^{R_{i}} a_{u}^{R_{j}}+\alpha_{C}^{R_{i}} a_{u}^{C}+\lambda_{u}^{S}\right) \mathrm{d} u} \\
& \times \int_{0}^{t} a_{u}^{R_{i}} e^{\int_{0}^{u}\left(\sum_{j \subset I \backslash\{i\}} \alpha_{R_{j}}^{R_{i}} a_{r}^{R_{j}}+\alpha_{C}^{R_{i}} a_{r}^{C}+\lambda_{r}^{S}\right) \mathrm{d} r} \\
& \times e^{-\int_{0}^{u}\left(\sum_{i \in I} a_{r}^{R_{i}}+a_{r}^{C}+\lambda_{r}^{S}\right) \mathrm{d} r} \mathrm{~d} u \\
= & e^{-\int_{0}^{t} \lambda_{u}^{S} \mathrm{~d} u} e^{-\int_{0}^{t}\left(\sum_{j \subset I \backslash\{i\}} \alpha_{R_{j}}^{R_{i}} a_{u}^{R_{j}}+\alpha_{C}^{R_{i}} a_{u}^{C}\right) \mathrm{d} u} \\
& \times \int_{0}^{t} a_{u}^{R_{i}} e^{\int_{0}^{u}\left(\sum_{j \subset I \backslash\{i\}} \alpha_{R_{j}}^{R_{i}} a_{r}^{R_{j}}+\alpha_{C}^{R_{i}} a_{r}^{C}\right) \mathrm{d} r} e^{-\int_{0}^{u}\left(\sum_{i \in I} a_{r}^{R_{i}}+a_{r}^{C}\right) \mathrm{d} r} \mathrm{~d} u .
\end{aligned}
$$

$P_{\mathrm{H}^{R_{M}}}\left(0, t \mid \psi^{S}\right)$ contains one random term $e^{-\int_{0}^{t} \lambda_{u}^{S} \mathrm{~d} u}$ outside the integral.

Assume that (i) holds for $|M|=k-1$. When $|M|=k$,

$$
\begin{aligned}
P_{\mathrm{H}^{R_{M}}}\left(0, t \mid \psi^{S}\right)= & e^{\int_{0}^{t} \Lambda_{\mathrm{H}^{R_{M}}}(u) \mathrm{d} u} \\
& \cdot \sum_{i \in M}\left[\int_{0}^{t}\left(\prod_{j \in M \backslash\{i\}} \alpha_{R_{i}}^{R_{j}}\right) a_{u}^{R_{i}} e^{-\int_{0}^{u} \Lambda_{\mathrm{H}^{R_{M}}}(r) \mathrm{d} r}\right. \\
& \left.\times P_{\mathrm{H}^{R_{M \backslash\{i\}}}}\left(0, u \mid \psi^{S}\right)\right] \mathrm{d} u .
\end{aligned}
$$

In the integral of (25), the only random term $e^{-\int_{0}^{u} \lambda_{r}^{S} \mathrm{~d} r}$ contained in $P_{\mathrm{H}^{R_{M \backslash\{i\}}}}\left(0, u \mid \psi^{S}\right)$ offsets the term $e^{\int_{0}^{u} \lambda_{r}^{S} \mathrm{~d} r}$ in $e^{-\int_{0}^{u} \Lambda_{\mathrm{H}^{R_{M}}}(r) \mathrm{d} r}$. Then, $P_{\mathrm{H}^{R_{M}}}\left(0, t \mid \psi^{S}\right)$ contains only one random term $e^{-\int_{0}^{t} \lambda_{u}^{s} \mathrm{~d} u}$ from $e^{\int_{0}^{t} \Lambda_{\mathrm{H}^{R} M}(u) \mathrm{d} u}$ outside the integral in (25), and (i) holds for $|M|=k$.

(ii) Firstly, $|M|=0$ :

$$
\begin{aligned}
& P_{\mathrm{H}^{S}}\left(0, t \mid \psi^{S}\right)=e^{\int_{0}^{t} \Lambda_{\mathrm{H}^{S}}(u) \mathrm{d} u} \int_{0}^{t} \lambda_{u}^{S} e^{-\int_{0}^{u} \Lambda_{\mathrm{H}^{S}}(r) \mathrm{d} r} P_{\mathrm{H}^{R_{\phi}}}\left(0, u \mid \psi^{S}\right) \mathrm{d} u
\end{aligned}
$$

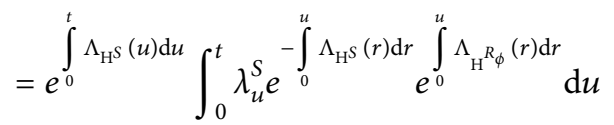

$$
\begin{aligned}
& =e^{\int_{0}^{t} \Lambda_{\mathrm{H}} S(u) \mathrm{d} u} \int_{0}^{t} \lambda_{u}^{S} e^{-\int_{0}^{u} \lambda_{r}^{S} \mathrm{~d} r} e^{-\int_{0}^{u} \Lambda_{\mathrm{H}}(r) \mathrm{d} r} e^{-\int_{0}^{u}\left(\sum_{i \in I} a_{r}^{R_{i}}+a_{r}^{C}\right) \mathrm{d} r} \mathrm{~d} u .
\end{aligned}
$$


$P_{\mathrm{H}^{S}}\left(0, t \mid \psi^{S}\right)$ contains one random term $\lambda_{u}^{S} e^{-\int_{0}^{u} \lambda_{r}^{S} \mathrm{~d} r}$ inside the integral.

Secondly, $|M|=1$ :

$$
\begin{aligned}
& P_{\mathrm{H}^{R_{\{i\}}} \cup s}\left(0, t \mid \psi^{S}\right)=e^{\int_{0}^{t} \Lambda_{\mathrm{H} R_{\{i\}} \cup S}(u) \mathrm{d} u}\left[\int_{0}^{t} \lambda_{u}^{S} e^{-\int_{0}^{u} \Lambda_{\mathrm{H}^{R_{i j}} \cup S(r) \mathrm{d} r}} P_{\mathrm{H}^{R_{\{i\}}}}\left(0, u \mid \psi^{S}\right) \mathrm{d} u+\int_{0}^{t} \alpha_{R_{i}}^{S} a_{u}^{R_{i}} e^{-\int_{0}^{u} \Lambda_{\mathrm{H}^{R_{i j}} \cup S}(r) \mathrm{d} r} P_{\mathrm{H}^{S}}\left(0, u \mid \psi^{S}\right) \mathrm{d} u\right]
\end{aligned}
$$

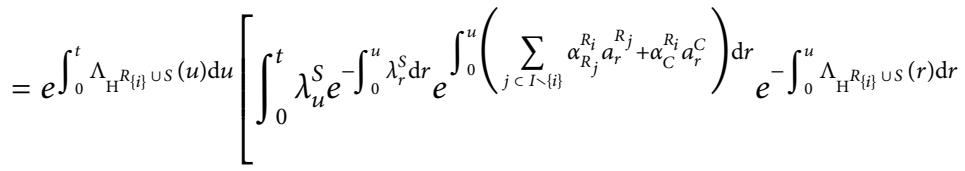

$$
\begin{aligned}
& \times \int_{0}^{u} a_{r}^{R_{i}} e^{-\int_{0}^{r}\left(\sum_{j \subset I \wedge\{i\}} \alpha_{R_{j}}^{R_{i}} a_{v}^{R_{j}}+\alpha_{C}^{R_{i}} a_{v}^{C}\right) \mathrm{d} v \int_{0}^{r}\left(\sum_{i \in I} a_{v}^{R_{i}}+a_{v}^{C}\right) \mathrm{d} v} \mathrm{~d} r \mathrm{~d} u \\
& +\int_{0}^{t} \alpha_{R_{i}}^{S} a_{u}^{R_{i}} e^{-\int_{0}^{u} \Lambda_{\mathrm{H}^{R}} R_{i j} \cup S(r) \mathrm{d} r} e^{\int_{0}^{u} \Lambda_{\mathrm{H}} S(r) \mathrm{d} r} \\
& \left.\times \int_{0}^{u} \lambda_{r}^{S} e^{-\int_{0}^{r} \lambda_{v}^{S} \mathrm{~d} v} e^{-\int_{0}^{r} \Lambda_{\mathrm{H}} S(v) \mathrm{d} v} e^{-\int_{0}^{r}\left(\sum_{i \in I} a_{v}^{R_{i}}+a_{v}^{C}\right) \mathrm{d} v} \mathrm{~d} r \mathrm{~d} u\right]
\end{aligned}
$$

The two terms in the right side of (27) contain only one Assume that (ii) holds for $|M|=k-1$. When $|M|=k$, random term $\lambda_{u}^{S} e^{-\int_{0}^{u} \lambda_{r}^{S} \mathrm{~d} r}$ (or $\lambda_{r}^{S} e^{-\int_{0}^{r} \lambda_{v}^{S} \mathrm{~d} v}$ ) inside the integral.

$$
\begin{aligned}
P_{\mathrm{H}^{R_{M}} \cup S}\left(0, t \mid \psi^{S}\right)= & e^{\int_{0}^{t} \Lambda_{\mathrm{H}^{R_{M}} \cup S}(u) \mathrm{d} u}\left\{\int_{0}^{t} \lambda_{u}^{S} e^{-\int_{0}^{u} \Lambda_{\mathrm{H}^{R_{M}} \cup S}(r) \mathrm{d} r} P_{\mathrm{H}^{R_{M}}}\left(0, u \mid \psi^{S}\right) \mathrm{d} u\right. \\
& \left.+\sum_{i \in M}\left[\alpha_{R_{i}}^{S}\left(\prod_{j \in M \backslash\{i\}} \alpha_{R_{i}}^{R_{j}}\right) \int_{0}^{t} a_{u}^{R_{i}} e^{-\int_{0}^{u} \Lambda_{\mathrm{H}^{R_{M}} \cup S}(r) \mathrm{d} r} \times P_{\mathrm{H}^{\left.R_{M} \backslash i\right\}}} \cup s\left(0, u \mid \psi^{S}\right) \mathrm{d} u\right]\right\} .
\end{aligned}
$$

By (i), $P_{\mathrm{H}^{R_{M}}}\left(0, u \mid \psi^{S}\right)$ only contains one random term $e^{-\int_{0}^{u} \lambda_{r}^{S} \mathrm{~d} r}$ which is outside the integral symbol. Then, the first term in $P_{\mathrm{H}^{R_{M}} \cup S}\left(0, t \mid \psi^{S}\right)$ contains only one random term $\lambda_{u}^{S} e^{-\int_{0}^{u} \lambda_{r}^{S} \mathrm{~d} r}$. By induction, each term in $P_{\mathrm{H}^{R_{M} \backslash\{i\}}} \cup S\left(0, t \mid \psi^{S}\right)$ contains only one random term $\lambda_{u}^{S} e^{-\int_{0}^{u} \lambda_{r}^{S} \mathrm{~d} r}$ which is in the integral symbol. Then, each term in the sum part of equation (28) contains only one random term $\lambda_{u}^{S} e^{-\int_{0}^{u} \lambda_{r}^{S} \mathrm{~d} r}$ in the integral. In summary, (ii) also holds when $|M|=k$. 
According to Proposition 1 and Fubini's theorem, we can take the expectation $E_{\psi^{s}}[\cdot]$ of equation (22) and obtain the unconditional transition probabilities as follows:

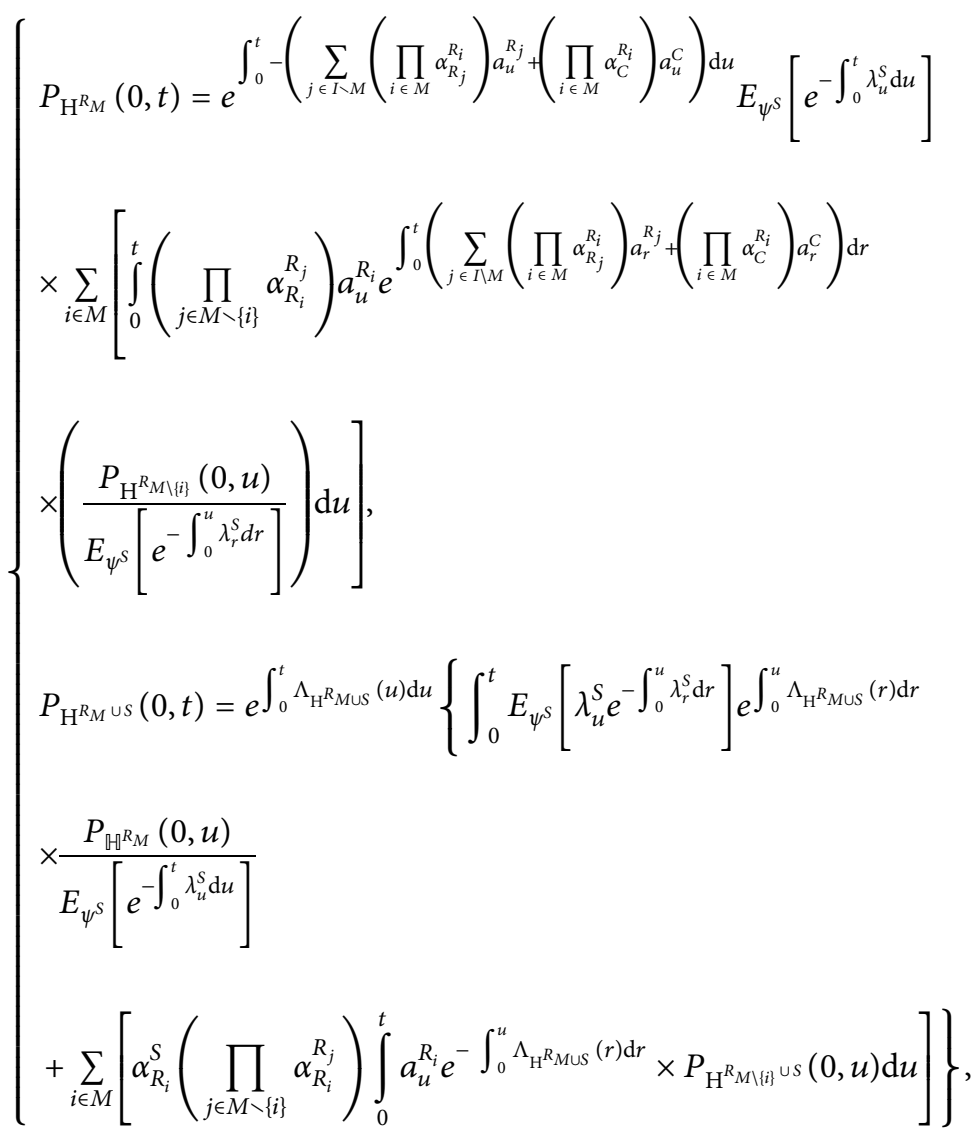

where $E_{\psi^{s}}[\cdot]$ is the expectation taken over the path of $\left(\lambda_{t}^{S}\right)_{t \in[0, T]}$.

To compute $E_{\psi^{s}}\left[e^{-\int_{0}^{t} \lambda_{u}^{S} \mathrm{~d} u}\right]$ and $E_{\psi^{S}}\left[\lambda_{t}^{S} e^{-\int_{0}^{t} \lambda_{u}^{S} \mathrm{~d} u}\right]$, we adopt the affine diffusion process with the jump for $\lambda_{t}^{S}$ as that proposed by Wang and Garleanu [5], which is a special Lévy process. We use Cox process to describe the external shock to obtain the closed-form solutions in this paper. The stochastic differential equation of $\lambda_{t}^{S}$ is given by

$$
\mathrm{d} \lambda_{t}^{S}=k\left(\theta-\lambda_{t}^{S}\right) \mathrm{d} t+\sigma \sqrt{\lambda_{t}^{S}} \mathrm{~d} Z_{t}+\Delta J_{t}
$$

where $Z_{t}$ is a standard Brownian motion, $J_{t}$ is a pure jump process, and $\Delta J_{t}$ denotes the jump of $J_{t}$ at time $t$. Here, $J_{t}$ is taken to be independent of $Z_{t}$ with jump sizes that are independent and exponentially distributed with mean $\mu$ and whose jump times are those of all independent Poisson processes with constant jump arrival rate $l$. It was shown by Wang and Garleanu [5] that

$$
\left\{\begin{array}{l}
E_{\psi^{S}}\left[e^{-\int_{0}^{t} \lambda_{u}^{S} d u}\right]=e^{\alpha(t)+\beta(t) \lambda_{0}^{S}}, \\
E_{\psi^{S}}\left[\lambda_{t}^{S} e^{-\int_{0}^{t} \lambda_{u}^{S} d u}\right]=-\left[\bar{\alpha}(t)+\bar{\beta}(t) \lambda_{0}^{S}\right] e^{\alpha(t)+\beta(t) \lambda_{0}^{S}},
\end{array}\right.
$$

where 


$$
\begin{aligned}
& \alpha(t)=\frac{k \theta\left(c_{1}+d_{1}\right)}{b_{1} c_{1} d_{1}} \ln \frac{c_{1}+d_{1} e^{b_{1} t}}{c_{1}+d_{1}}+\frac{k \theta}{c_{1}} t \\
& +\frac{l\left(a_{2} c_{2}-d_{2}\right)}{b_{2} c_{2} d_{2}} \ln \frac{c_{2}+d_{2} e^{b_{2} t}}{c_{2}+d_{2}}+\left(\frac{l}{c_{2}}-l\right) t, \\
& \beta(t)=\frac{1-e^{b_{1} t}}{c_{1}+d_{1} e^{b_{1} t}}, \\
& \bar{\alpha}(t)=\frac{k \theta\left(c_{1}+d_{1}\right)}{c_{1}} \frac{e^{b_{1} t}}{c_{1}+d_{1} e^{b_{1} t}}+\frac{k \theta}{c_{1}} \\
& +\frac{l\left(a_{2} c_{2}-d_{2}\right)}{c_{2}} \frac{e^{b_{2} t}}{c_{2}+d_{2} e^{b_{2} t}}+\left(\frac{l}{c_{2}}-l\right) \\
& \bar{\beta}(t)=\frac{-\left(k^{2}+2 \sigma^{2}\right) e^{b_{1} t}}{\left(c_{1}+d_{1} e^{b_{1} t}\right)^{2}}, \\
& b_{1}=-\sqrt{k^{2}+2 \sigma^{2}} \text {, } \\
& c_{1}=\frac{k+\sqrt{k^{2}+2 \sigma^{2}}}{-2}, \\
& d_{1}=\frac{-k+\sqrt{k^{2}+2 \sigma^{2}}}{-2} \\
& a_{2}=\frac{-k+\sqrt{k^{2}+2 \sigma^{2}}}{k+\sqrt{k^{2}+2 \sigma^{2}}}, \\
& b_{2}=b_{1}, d_{2}=\frac{d_{1}+\mu}{c_{1}}, \\
& c_{2}=1-\frac{\mu}{c_{1}} .
\end{aligned}
$$

Replacing the conditional expectations in (29) with equation (31), we obtain the unconditional transition probabilities.

\section{Pricing Basket Credit Derivatives}

We will compute the fair premium rates of BCDS and BCLN with the counterparty risk in this section. Under the continuous model assumption, the premium rates are paid continuously at a constant rate. We assume that the notional of the BCDS or BCLN is $1, T$ is the maturity date of the contracts, $c_{k}$ is the premium rate of $k$ th-to-default credit derivatives, $\tau_{k}$ is the $k$ th default time of reference entities, constant $r$ is the risk-free interest rate, and constant $\rho \in[0,1)$ is the recovery rate.

3.1. $k$ th-to-Default CDS. The cash flow of a $k$ th-to-default CDS is presented in Figure 1.

(i) The protection buyer pays constant premium rate $c_{k}$ during the life of the $k$ th-to-default CDS (ii) If the $k$ th default in the pool occurs before the maturity, the protection buyer gives a recovery payoff $\rho$, and the contract is terminated

The expected cash flow of $k$ th-to-default CDS is as follows (see Zheng and Jiang [9]):

$$
E\left[\int_{0}^{T} c_{k} e^{-r s} I_{\tau_{k}>s} I_{\tau_{C}>s} \mathrm{~d} s\right]=E\left[(1-\rho) e^{-r \tau_{k}} I_{\tau_{k} \leq T} I_{\tau_{C}>T}\right],
$$

and the premium rate is given by

$$
c_{k}=\frac{E\left[(1-\rho) e^{-r \tau_{k}} I_{\tau_{k} \leq T} I_{\tau_{C}>T}\right]}{E\left[\int_{0}^{T} e^{-r s} I_{\tau_{k}>s} I_{\tau_{C}>s} \mathrm{~d} s\right]} .
$$

To calculate the premium rate for the $k$ th-to-default CDS, there are two possible scenarios during $[0, t]$ : nonoccurrence of the external shock $S$ or occurrence of $S$. If $k-1$ reference entities have defaulted during $[0, t]$ and another reference entity defaults during $(t, t+\mathrm{d} t]$, the probability of such occurrence is given by

$$
\begin{aligned}
Q_{k}(t) \mathrm{d} t= & \sum_{M \subset I,|M|=k-1}\left[P_{\mathrm{H}^{R_{M}}}(0, t)\left(\sum_{j \in I \backslash M}\left(\prod_{i \in M} \alpha_{R_{j}}^{R_{i}}\right) a_{t}^{R_{j}}\right)\right. \\
& \left.+P_{\mathrm{H}^{R_{M}} \cup S}(0, t) \times\left(\sum_{j \subset I \backslash M} \alpha_{R_{j}}^{S}\left(\prod_{i \in M} \alpha_{R_{j}}^{R_{i}}\right) a_{t}^{R_{j}}\right)\right] \mathrm{d} t,
\end{aligned}
$$

where $\quad \sum_{M \subset I,|M|=k-1}\left[P_{\mathrm{H}^{R_{M}}}(0, t)\left(\sum_{j \in I \backslash M}\left(\prod_{i \in M} \alpha_{R_{j}}^{R_{i}}\right) a_{t}^{R_{j}}\right)\right] \mathrm{d} t$ corresponds to nonoccurrence of $S$ and $\sum_{M \subset I,|M|=k-1}\left[P_{\mathbf{H}^{R_{M}} \cup S}(0, t) \times\left(\sum_{j \subset I \backslash M} \alpha_{R_{j}}^{S}\left(\prod_{i \in M} \alpha_{R_{j}}^{R_{i}}\right) a_{t}^{R_{j}}\right)\right] \mathrm{d} t$ corresponds to the case otherwise. So, the expected present value of the compensation payment paid by the protection seller within $(t, t+\mathrm{d} t]$ is

$$
(1-\rho) e^{-r t} Q_{k}(t) \mathrm{d} t
$$

Over the entire period $[0, T]$, the expected present value of the compensation payment paid by the protection seller is

$$
E\left[(1-\rho) e^{-r \tau_{k}} I_{\tau_{k} \leq T} I_{\tau_{C}>T}\right]=\int_{0}^{T}(1-\rho) e^{-r t} Q_{k}(t) \mathrm{d} t .
$$

The $k$ th-to-default CDS contract will be terminated if more than $k-1$ reference entities default. The corresponding states to continue the contract are $\mathrm{H}^{R_{M}}$ and $\mathrm{H}^{R_{M}} \cup S(M \subset I, 0 \leq|M| \leq k-1)$. The expected premium payment paid by the protection buyer within $(t, t+\mathrm{d} t]$ is given by

$$
c_{k} e^{-r t} D_{k}(t) \mathrm{d} t
$$

where

$$
D_{k}(t)=\sum_{M \subset I, 0 \leq|M| \leq k-1}\left[P_{\mathrm{H}^{R_{M}}}(0, t)+P_{\mathrm{H}^{R_{M}} \cup S}(0, t)\right] .
$$




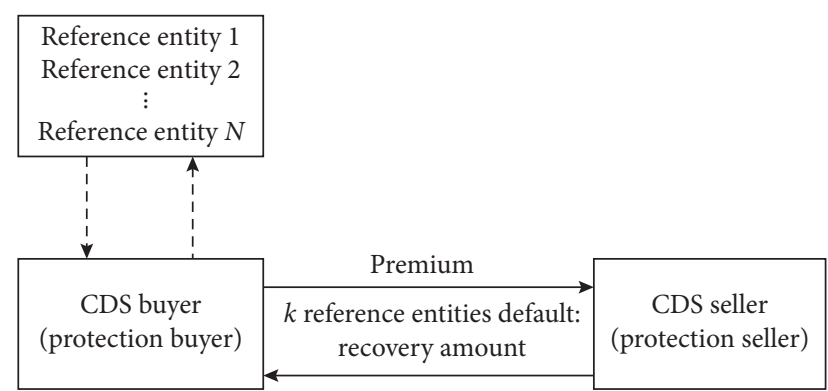

Figure 1: The cash flow of $k$ th-to-default CDS.

Over the entire period $[0, T]$, the expected present value of the premium payment paid by the protection buyer is

$$
E\left[\int_{0}^{T} c_{k} e^{-r s} I_{\tau_{k}>s} I_{\tau_{C}>s} \mathrm{~d} s\right]=c_{k} \int_{0}^{T} e^{-r t} D_{k}(t) \mathrm{d} t .
$$

By (34), the $k$ th-to-default CDS premium rate is given by

$$
c_{k}=(1-\rho) \frac{\int_{0}^{T} e^{-r t} Q_{k}(t) \mathrm{d} t}{\int_{0}^{T} e^{-r t} D_{k}(t) \mathrm{d} t} .
$$

3.2. kth-to-Default $C L N$. The cash flow of a $k$ th-to-default CLN is presented in Figure 2.

(i) The protection seller pays a nominal amount at the initial date

(ii) The protection buyer pays constant premium rate $c_{k}$ during the life of the $k$ th-to-default CLN

(iii) If the $k$ th default in the pool occurs before the maturity, the protection buyer gives a recovery payoff $\rho$, and the contract is terminated

(iv) If the $k$ th default in the pool does not occur before the maturity, the protection buyer returns the nominal amount follows:

The expected cash flow of the $k$ th-to-default CLN is as

$$
\begin{aligned}
1= & E\left[\int_{0}^{T} c_{k} e^{-r s} I_{\tau_{k}>s} I_{\tau_{C}>s} \mathrm{~d} s+\rho e^{-r \tau_{k}} I_{\tau_{k} \leq T} I_{\tau_{C}>T}\right. \\
& \left.+e^{-r T} I_{\tau_{k}>T} I_{\tau_{C}>T}\right] .
\end{aligned}
$$

The premium rate is given by

$$
c_{k}=\frac{E\left[1-\rho e^{-r \tau_{k}} I_{\tau_{k} \leq T} I_{\tau_{C}>T}-e^{-r T} I_{\tau_{k}>T} I_{\tau_{C}>T}\right]}{E\left[\int_{0}^{T} e^{-r s} I_{\tau_{k}>s} I_{\tau_{C}>s} \mathrm{~d} s\right]} .
$$

Here, the method for calculating the default probabilities is the same as the method for the $k$ th-to-default CDS case.

Over the whole period $[0, T]$, the expected present value of the recovery payment paid by the CLN issuer is

$$
E\left[\rho e^{-r \tau_{k}} I_{\tau_{k} \leq T} I_{\tau_{C}>T}\right]=\int_{0}^{T} \rho e^{-r t} Q_{k}(t) \mathrm{d} t,
$$

where

$$
\begin{aligned}
Q_{k}(t) \mathrm{d} t= & \sum_{M \subset I,|M|=k-1}\left[P_{\mathrm{H}^{R_{M}}}(0, t)\left(\sum_{j \in I \backslash M}\left(\prod_{i \in M} \alpha_{R_{j}}^{R_{i}}\right) a_{t}^{R_{j}}\right)\right. \\
& \left.+P_{\mathrm{H}^{R_{M}} \cup \mathrm{S}}(0, t) \times\left(\sum_{j \subset I \backslash M} \alpha_{R_{j}}^{S}\left(\prod_{i \in M} \alpha_{R_{j}}^{R_{i}}\right) a_{t}^{R_{j}}\right)\right] \mathrm{d} t .
\end{aligned}
$$

The expected present value of the premium payment paid by the CLN issuer is

$$
E\left[\int_{0}^{T} c_{k} e^{-r s} I_{\tau_{k}>s} I_{\tau_{C}>s} \mathrm{~d} s\right]=c_{k} \int_{0}^{T} e^{-r t} D_{k}(t) \mathrm{d} t,
$$

where

$$
D_{k}(t)=\sum_{M \subset I, 0 \leq|M| \leq k-1}\left[P_{\mathrm{H}^{R_{M}}}(0, t)+P_{\mathrm{H}^{R_{M}} \cup \mathcal{S}}(0, t)\right] .
$$

The expectation of the payment of nominal amount at maturity of the contract is

$$
E\left[e^{-\mathrm{rT}} I_{\tau_{k}>T} I_{\tau_{C}>T}\right]=e^{-\mathrm{rT}} D_{k}(T) .
$$

By (43), the kth-to-default CLN premium rate is given by

$$
c_{k}=\frac{1-\rho \int_{0}^{T} e^{-\mathrm{rt}} Q_{k}(t) \mathrm{d} t-e^{-\mathrm{rT}} D_{k}(T)}{\int_{0}^{T} e^{-\mathrm{rt}} D_{k}(t) \mathrm{d} t} .
$$

\section{Numerical Analysis}

In this section, we perform numerical experiments to show how the correlated default risk may affect the premium rates. We will investigate the sensitivity of the premium rates to the parameters of our model.

4.1. kth-to-Default CDS. The parameters of the model are assumed to be 


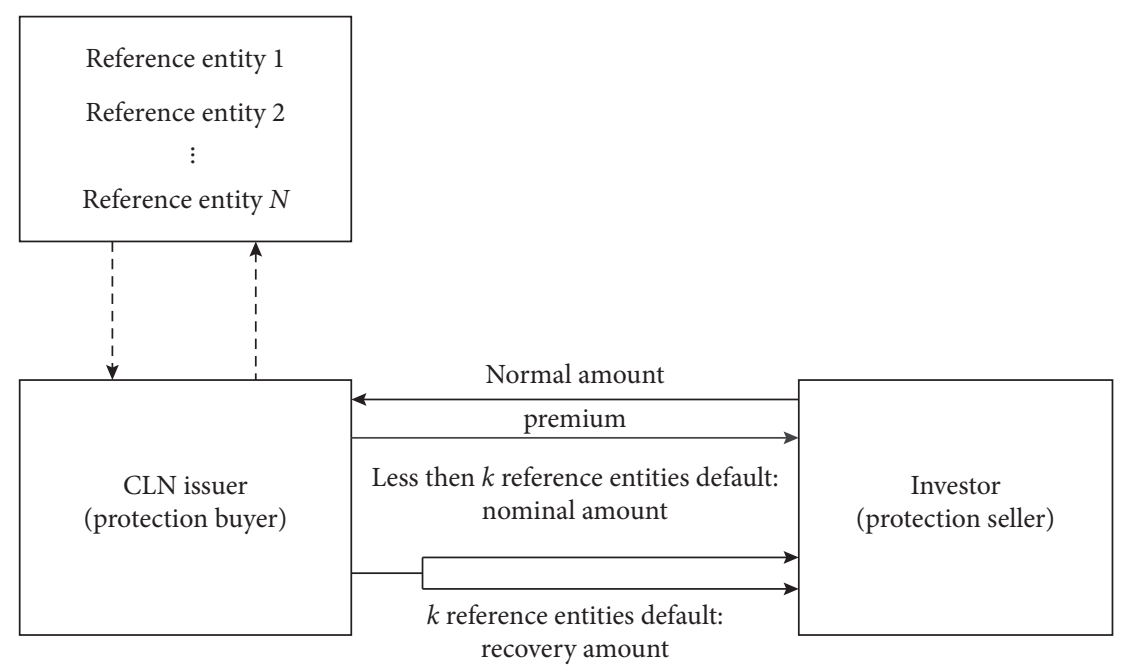

Figure 2: The cash flow of the $k$ th-to-default CLN.

$$
\begin{aligned}
\rho & =0.4, \\
r & =0.04, \\
\alpha_{R_{i}}^{S} & =1.1, \\
\alpha_{C}^{S} & =1.1, \\
\alpha_{R_{j}}^{R_{i}} & =1.1, \\
\alpha_{C}^{R_{i}} & =1.1, \\
\lambda_{0}^{S} & =0.05, \\
\sigma & =0.2, \\
k & =0.3, \\
\theta & =0.02, \\
l & =0.3, \\
\mu & =0.15, \\
T & =5 .
\end{aligned}
$$

For convenience of calculation, we assume

$$
a_{R_{i}}(t)=a_{C}(t)=0.1
$$

We take a basket CDS with ten reference entities, for example. Table 1 lists the $k$ th-to-default CDS premium rates for $k=1, \ldots, 10$.

The CDS premium rates decrease as $k$ increases because the default probability of $k$ reference entities decreases with increasing of $k$. Lower default probability leads to lower premium rates. The premium rates tend to 0 with increasing of $k$.

We analyze the effects of the default correlation on $k$ thto-default CDS premium rates for $k=1,4$.

The numerical calculation shows that the defaults of other reference entities have no effects on the 1st-to-default CDS. The premium rates of $k$ th-to-default CDS have similar
TABle 1: The premium rates of the $k$ th-to-default CDS.

\begin{tabular}{lc}
\hline$k$ th & Premium rate $(\%)$ \\
\hline 1st & 60.3724 \\
2nd & 28.7716 \\
3rd & 17.2977 \\
4 th & 13.5195 \\
th & 8.3919 \\
6th & 4.4503 \\
7th & 1.8347 \\
8th & 0.4272 \\
9th & 0.0565 \\
10th & 0.0000009 \\
\hline
\end{tabular}

trends on the default correlation for $k=2,3, \ldots, 10$. We just choose immediate number 4 to illustrate the trends.

Figure 3 illustrates that $k$ th-to-default CDS premium rates are increasing functions of $\alpha_{R_{i}}^{S}(i \in I)$ because the reference entities become more risky with higher $\alpha_{R_{i}}^{S}$, and a higher default risk leads to a higher premium rate.

Figure 4 illustrates that $k$ th-to-default CDS premium rates are decreasing functions of $\alpha_{C}^{S}$ because the counterparty becomes more risky with higher $\alpha_{C}^{S}$, and a higher counterparty risk leads to a lower premium rate.

Figure 5 illustrates that $k$ th-to-default CDS $(k \geq 2)$ premium rates are increasing functions of $\alpha_{R_{j}}^{R_{i}}(i \in I, j \in I \backslash\{i\})$ because the reference entities become more risky with high $\alpha_{R_{j}}^{R_{i}}$, and a higher default risk leads to a higher premium rate. The contagion risk has no effect on 1th-to-default CDS because the contract will be terminated if any reference entity defaulted.

Figure 6 illustrates that $k$ th-to-default CDS $(k \geq 2)$ premium rates are decreasing functions of $\alpha_{C}^{R_{i}}(i \in I)$ because the counterparty becomes more risky with high $\alpha_{C}^{R_{i}}$, and a higher counterparty risk leads to a lower premium rate. The contagion risk has no effect on 1st-to-default CDS because the contract will be terminated if any reference entity defaulted. 


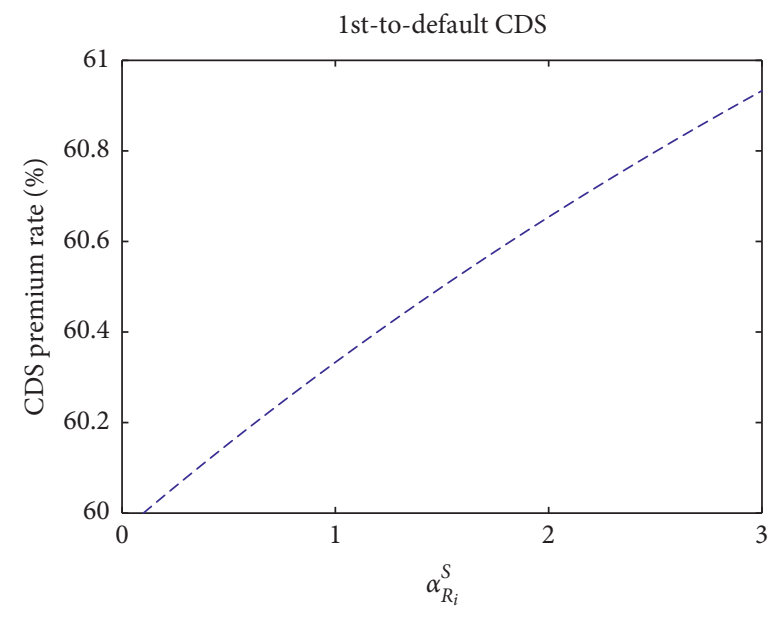

(a)

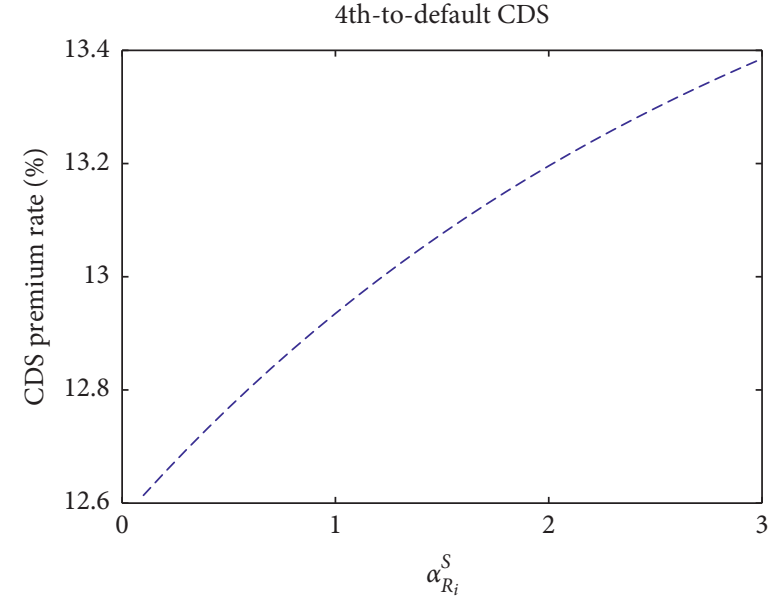

(b)

FIgURE 3: The premium rates of the $k$ th-to-default CDS.

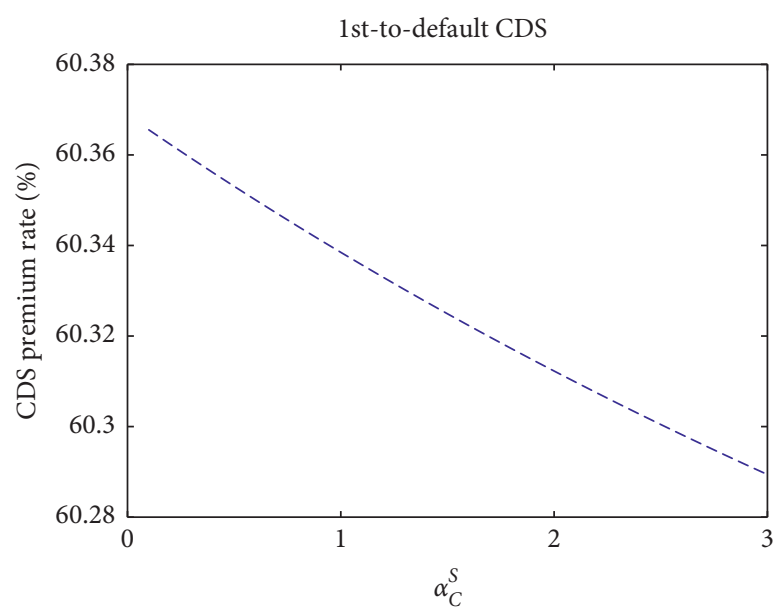

(a)

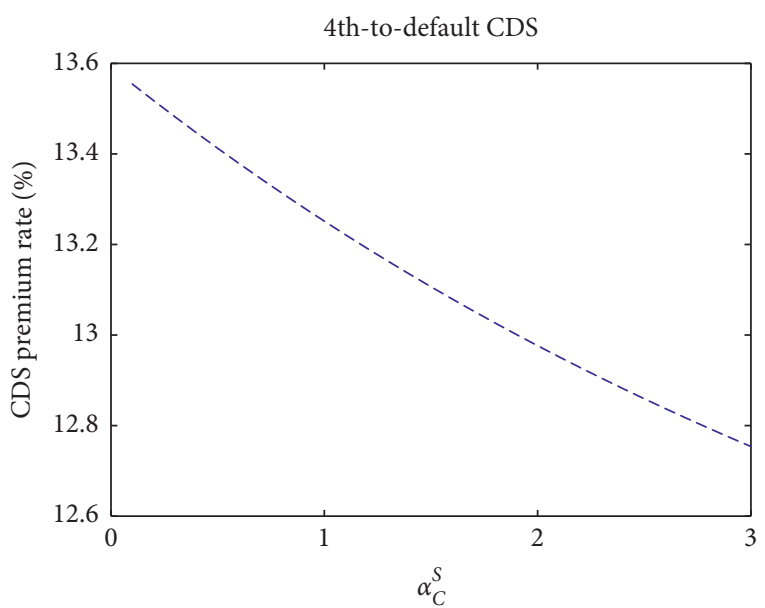

(b)

FIgURE 4: The premium rates of the $k$ th-to-default CDS.

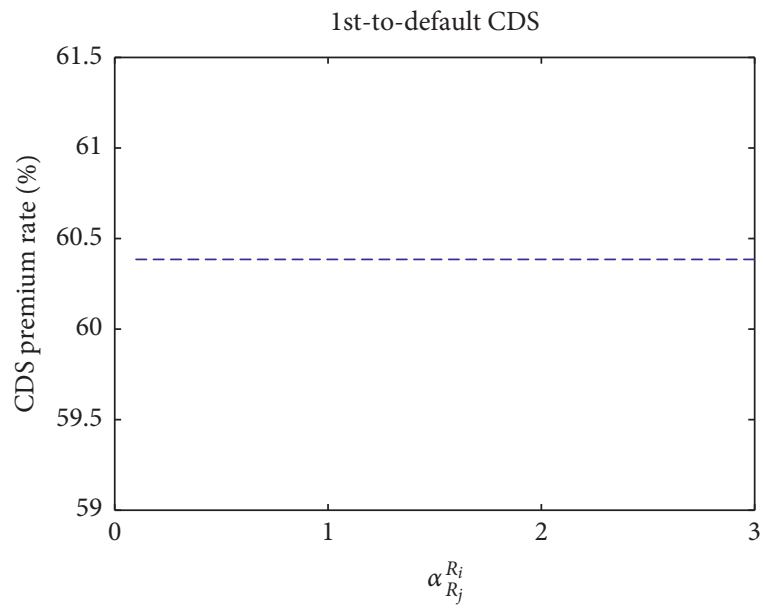

(a)

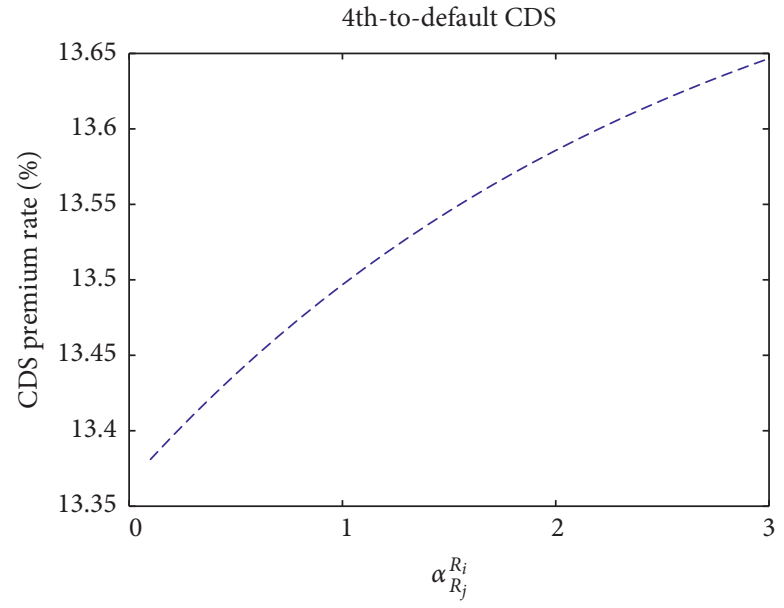

(b)

FIgUre 5: The premium rates of the $k$ th-to-default CDS. 
1st-to-default CDS

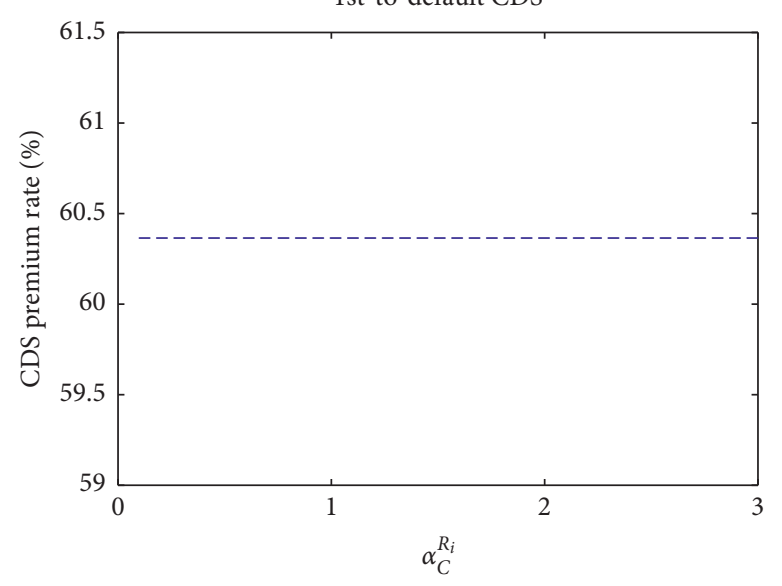

(a)

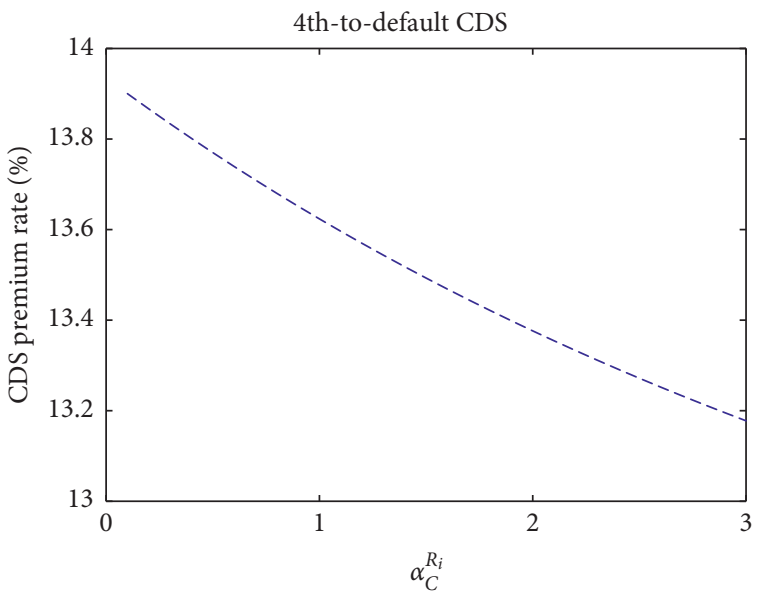

(b)

Figure 6: The premium rates of the $k$ th-to-default CDS.

TABLe 2: The premium rates of the $k$ th-to-default CLN.

\begin{tabular}{lr}
\hline$k$ th & Premium rate $(\%)$ \\
\hline 1st & 64.9980 \\
2nd & 33.4078 \\
3rd & 21.3288 \\
4th & 17.3264 \\
th & 12.6668 \\
6th & 8.4503 \\
7th & 5.8347 \\
8th & 4.9583 \\
9th & 4.0034 \\
10 th & 4.00000853 \\
\hline
\end{tabular}

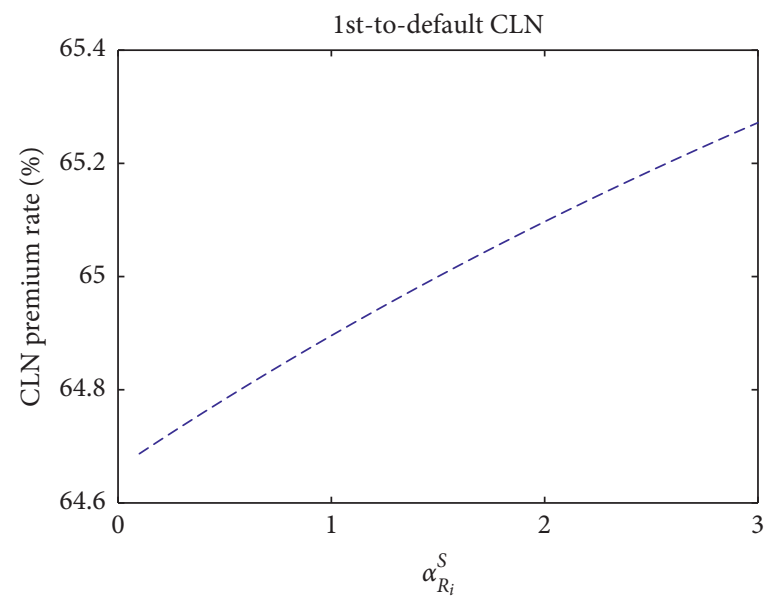

(a)

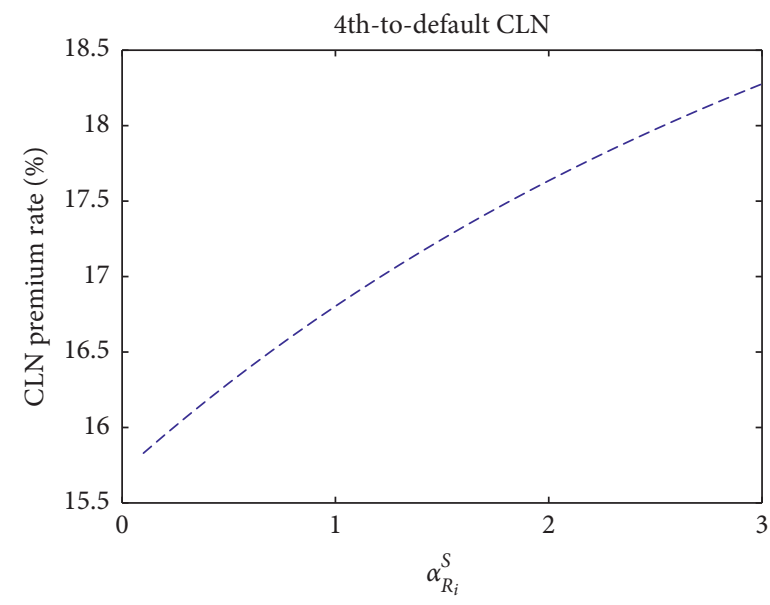

(b)

Figure 7: The premium rates of the $k$ th-to-default CLN. 


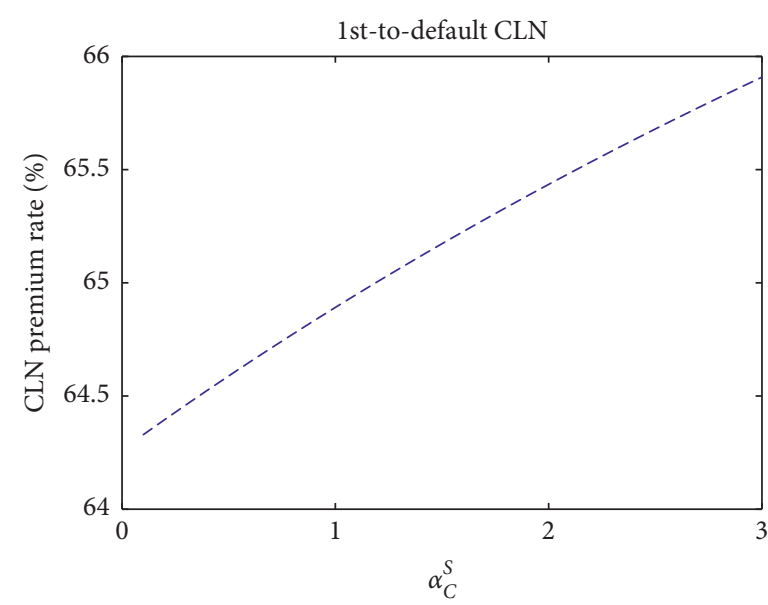

(a)

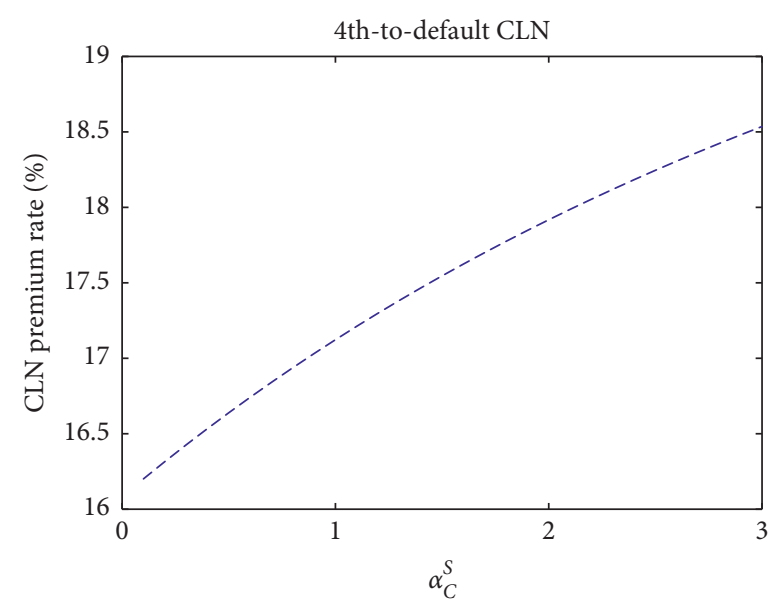

(b)

Figure 8: The premium rates of the $k$ th-to-default CLN.

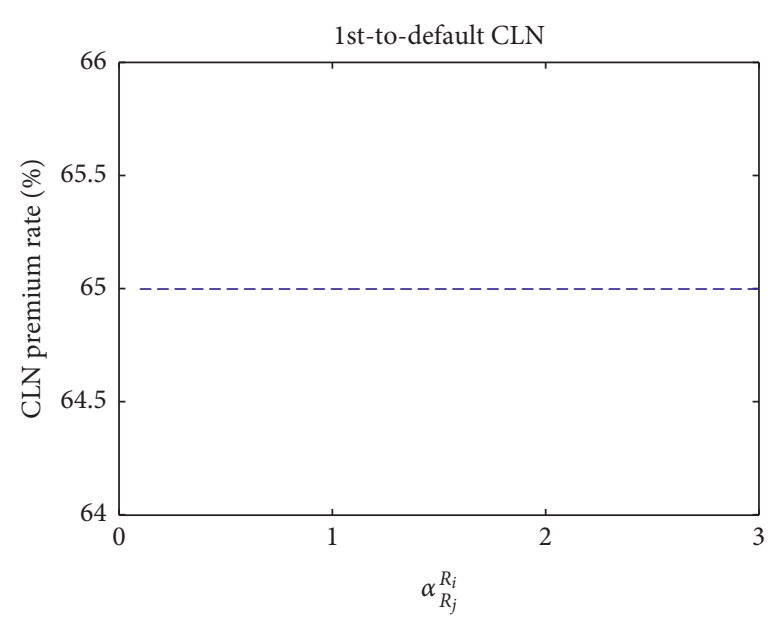

(a)

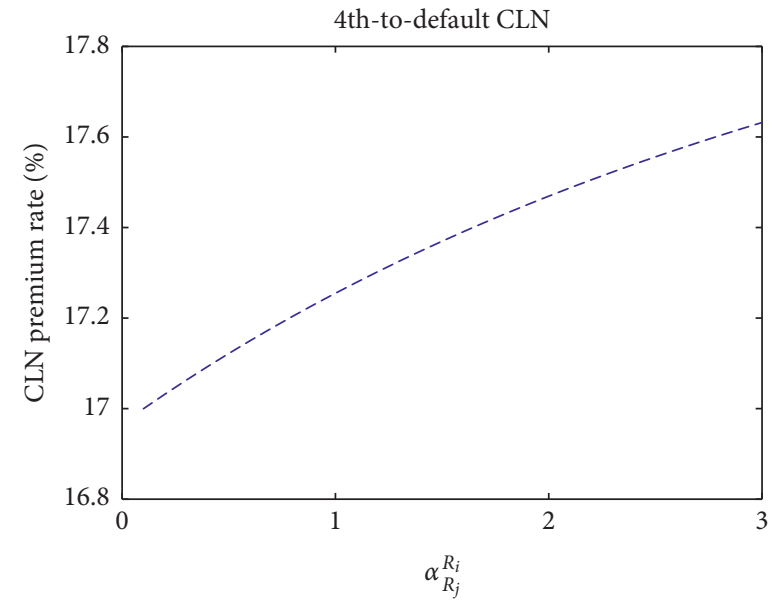

(b)

FIgURE 9: The premium rates of the $k$ th-to-default CLN.

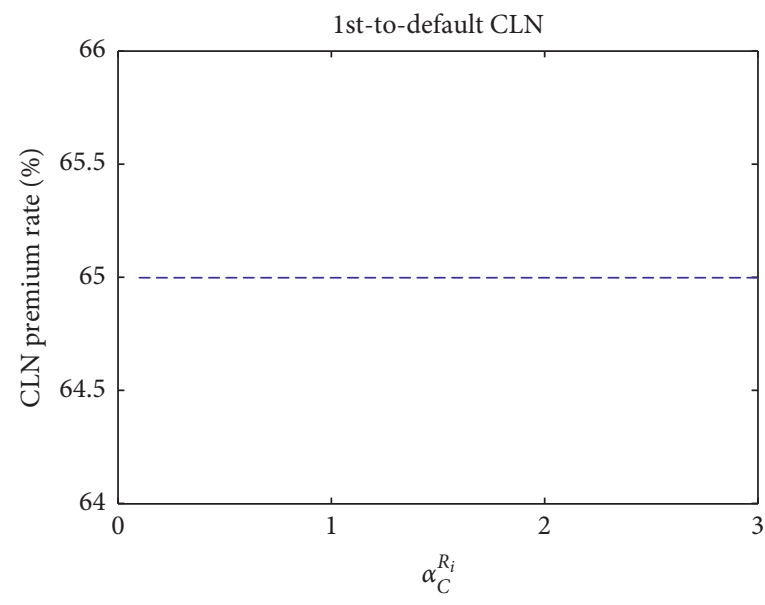

(a)

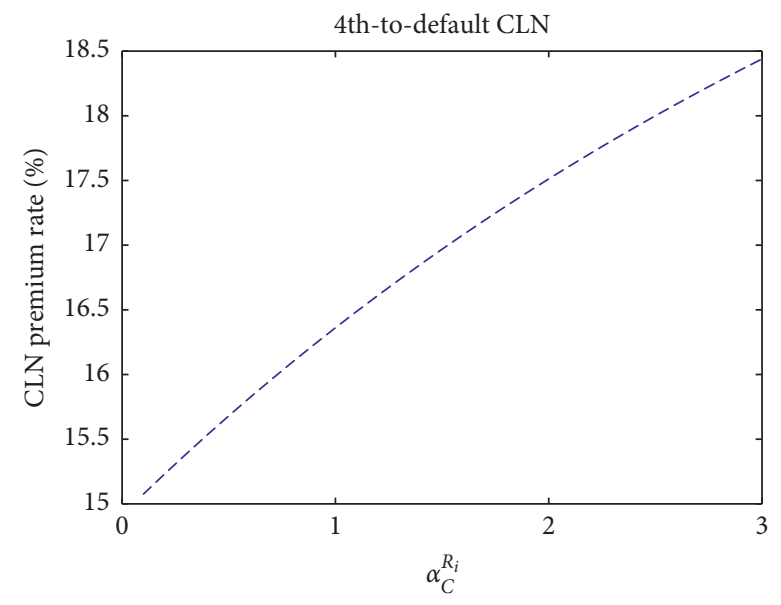

(b)

Figure 10: The premium rates of the $k$ th-to-default CLN. 
4.2. kth-to-Default CLN. The parameters of the model are assumed to be

$$
\begin{aligned}
\rho & =0.6, \\
r & =0.04, \\
\alpha_{R_{i}}^{S} & =1.1, \\
\alpha_{C}^{S} & =1.1, \\
\alpha_{R_{j}}^{R_{i}} & =1.1, \\
\alpha_{C}^{R_{i}} & =1.1, \\
\lambda_{0}^{S} & =0.05, \\
\sigma & =0.2, \\
k & =0.3, \\
\theta & =0.02, \\
l & =0.3, \\
\mu & =0.15, \\
T & =5 .
\end{aligned}
$$

For convenience of calculation, we assume

$$
a_{R_{i}}(t)=a_{C}(t)=0.1 \text {. }
$$

We take a basket CLN with ten reference entities, for example. Table 2 lists the $k$ th-to-default CLN premium rates for $k=1, \ldots, 10$.

The premium rates of the BCLN on $k$ have similar trends as the premium rate of BCDS. However, the premium rates tend to the risk-free interest rate with the increase of $k$.

The effects of the default correlation on $k$ th-to-default CLN premium rates are demonstrated for $k=1,4$. The numerical calculation shows that the defaults of other reference entities have no effects on the 1st-to-default CLN. The premium rates of the $k$ th-to-default CLN have similar trends on the default correlation for $k=2,3, \ldots, 10$. We just choose immediate number 4 to illustrate the trends.

Figure 7 illustrates that the premium rates of BCLN are increasing functions of $\alpha_{R_{i}}^{S}(i \in I)$, which is the same as the BCDS case.

Figure 8 illustrates that $k$ th-to-default CLN premium rates are increasing functions of $\alpha_{C}^{S}$ which is different from the BCDS case because the counterparty in the BCLN is the protection buyer.

Figure 9 illustrates that the $k$ th-to-default $(k \geq 2)$ CLN premium rates are increasing functions of $\alpha_{R_{j}}^{R_{i}}(i \in I, j \in I \backslash\{i\})$, which is the same as the BCDS case. The contagion risk also has no effect on the 1st-to-default CLN.

Figure 10 illustrates that $k$ th-to-default $(k \geq 2)$ CLN premium rates are increasing functions of $\alpha_{C}^{R_{i}}(i \in I)$, which is different from the BCDS case because the counterparty in the BCLN is the protection buyer. The contagion risk also has no effect on the 1st-to-default CLN.

\section{Conclusion}

In this paper, we use a Markov chain model with interacting intensities to compute the $k$ th-to-default CDS and $k$ th-todefault CLN premium rates. We model the default correlation among the names in the portfolio by an external shock as well as the defaults of other names in the portfolio. By presenting a general infinitesimal generator matrix of $2^{N+2} \times$ $2^{N+2}$ dimension, we obtain the joint distribution of default times. Then, the formulas of $k$ th-to-default CDS and $k$ th-todefault CLN premium rates are calculated. In numerical analysis, we show that the premium rates decrease as $k$ increases, and the contagion risk does not affect the 1st-todefault premium rate. Our numerical results also show the effects of correlated risks between the counterparty and reference entities on the premium rates. Due to different product structures between BCDS and BCLN, the impacts of the contagion risk on premium rates are different.

\section{Data Availability}

The data in this paper can be used publicly.

\section{Conflicts of Interest}

The authors declare that they have no conflicts of interest.

\section{Acknowledgments}

This work was supported by the National Natural Science Foundation of China (nos. 11671291 and 11901420) and Humanities and Social Science Research Projects in the Ministry of Education (no. 20YJAZH025).

\section{References}

[1] D. X. Li, "On default correlation," The Journal of Fixed Income, vol. 9, no. 4, pp. 43-54, 2000.

[2] P. Schőnbucher and D. Schubert, "Copula-dependent defaults in intensity models," SSRN Electronic Journal, vol. 63, Article ID 301968, 2001.

[3] D. Brigo and A. Capponi, "Bilateral counterparty risk valuation with stochastic dynamical models and application to credit default swaps," Cornell University, Ithaca, NY, USA, 2008, http://arxiv.org/abs/0812.3705.

[4] F. Jean-David, "Recent developments in copula models," Econometrics, vol. 5, no. 3, pp. 34-43, 2017.

[5] D. Wang and N. Gârleanu, "Risk and valuation of collateralized debt obligations," Financial Analysts Journal, vol. 57, no. 1, pp. 41-59, 2001.

[6] K. Giesecke, "A simple exponential model for dependent defaults," The Journal of Fixed Income, vol. 13, no. 3, pp. 74-83, 2003.

[7] X. Liang, G. Wang, and H. Li, "Pricing credit default swaps with bilateral counterparty risk in a reduced form model with Markov regime switching," Applied Mathematics and Computation, vol. 230, pp. 290-302, 2014.

[8] R. A. Jarrow and F. Yu, "Counterparty risk and the pricing of defaultable securities," The Journal of Finance, vol. 56, no. 5, pp. 1765-1799, 2001. 
[9] H. Zheng and L. Jiang, "Basket cds pricing with interacting intensities," Finance and Stochastics, vol. 13, no. 3, pp. 445-469, 2009.

[10] Y. Dong and G. Wang, "Bilateral counterparty risk valuation for credit default swap in a contagion model using Markov chain," Economic Modelling, vol. 40, no. 2, pp. 91-100, 2014.

[11] J. C. Hull and A. D. White, "Valuation of a CDO and an $\mathrm{n}$-th to default CDS without Monte Carlo simulation," The Journal of Derivatives, vol. 12, no. 2, pp. 8-23, 2004.

[12] M. B. Walker, "Credit default swaps with counterparty risk: a calibrated markov model," Journal of Credit Risk, vol. 2, no. 1, pp. 24-39, 2006.

[13] F. Yu, "Correlated defaults in intensity-based models," Mathematical Finance, vol. 17, no. 2, pp. 155-173, 2007.

[14] R. Frey and J. Backhaus, "Pricing and hedging of portfolio credit derivatives with interacting default intensities," International Journal of Theoretical and Applied Finance, vol. 11, no. 6, pp. 611-634, 2008.

[15] P.-C. Wu, "Applying a factor copula to value basket credit linked notes with issuer default risk," Finance Research Letters, vol. 7, no. 3, pp. 178-183, 2010.

[16] A. Herbertsson, J. Jang, and T. Schmidt, "Pricing basket default swaps in a tractable shot noise model," Statistics \& Probability Letters, vol. 81, no. 8, pp. 1196-1207, 2011.

[17] T. Wang, J. Liang, and X. Yang, "Pricing for basket cds and lcds," Modern Economy, vol. 03, no. 02, pp. 171-178, 2012.

[18] P. Li and Z.-Z. Li, "Change analysis for the dependence structure and dynamic pricing of basket default swaps," European Financial Management, vol. 21, no. 4, pp. 646-671, 2013.

[19] A. Esfahanipour and R. Jahanbin, “An application of genetic network programming model for pricing of basket default swaps (bds)," AUT Journal of Modeling and Simulation, vol. 49, no. 1, pp. 13-22, 2017.

[20] Y. Dong, K. C. Yuen, and K. Chuen Yuen, "Pricing credit derivatives under a correlated regime-switching hazard processes model," Journal of Industrial \& Management Optimization, vol. 13, no. 3, p. 1395, 2017.

[21] J. Guo, Y. Dong, and G. Wang, "Basket CDS pricing with default intensities using a regime-switching shot-noise model," Communications in Statistics - Theory and Methods, vol. 47, no. 18, pp. 4443-4458, 2018.

[22] K. S. Leung and Y. K. Kwok, "Counterparty risk for credit default swaps: Markov chain interacting intensities model with stochastic intensity," Asia-Pacific Financial Markets, vol. 16, no. 3, pp. 169-181, 2009. 